\title{
Target-oriented Gaussian beam migration using a modified ray tracing scheme
}

\author{
Rui Zhang ${ }^{1,2} \cdot$ Jian-Ping Huang ${ }^{1} \cdot$ Su-Bin Zhuang ${ }^{1} \cdot{\text { Zhen-Chun } \mathrm{Li}^{1}}^{1}$
}

Received: 2 December 2018 / Published online: 13 November 2019

(c) The Author(s) 2019

\begin{abstract}
For large-scale 3D seismic data, target-oriented reservoir imaging is more attractive than conventional full-volume migration, in terms of computation efficiency. Gaussian beam migration (GBM) is one of the most robust depth imaging method, which not only keeps the advantages of ray methods, such as high efficiency and flexibility, but also allows us to solve caustics and multipathing problems. But conventional Gaussian beam migration requires slant stack for prestack data, and ray tracing from beam center location to subsurface, which is not easy to be directly applied for target-oriented imaging. In this paper, we modify the conventional Gaussian beam migration scheme, by shooting rays from subsurface image points to receivers to implement wavefield back-propagation. This modification helps us to achieve a better subsurface illumination in complex structure and allows simple implementation for target reservoir imaging. Significantly, compared with the wavefield-based GBM, our method does not reconstruct the subsurface snapshots, which has higher efficiency. But the proposed method is not as efficient as the conventional Gaussian beam migration. Synthetic and field data examples demonstrate the validity and the target-oriented imaging capability of our method.
\end{abstract}

Keywords Gaussian beam migration $\cdot$ Target-oriented imaging $\cdot$ Illumination $\cdot$ Ray tracing

\section{Introduction}

Gaussian beam method started its successful path in geophysics in the early 1980s (Popov 1981; Kachalov and Popov 1981; Červený et al. 1982; Popov 1982; Červený 1983; Červený and Pšenčík 1983a, b, 1984). Its reliance on ray theory enables it to keep the advantages of ray-based

Edited by Jie Hao

Jian-Ping Huang

jphuang@upc.edu.cn

Rui Zhang

zhangruixiaoz@163.com

Su-Bin Zhuang

subin_zhuang@163.com

Zhen-Chun Li

leonli@upc.edu.cn

1 School of Geosciences, China University of Petroleum (East China), Qingdao 266580, China

2 State Key Laboratory of Oil and Gas Reservoir Geology and Exploitation, Southwest Petroleum University, Chengdu 610500, China methods. For instance, it provides physical insight of the propagation of the seismic wave, which makes it easier for us to describe the wave phenomena in quite complicated geophysical models. In addition, it is more efficient than wave equation migration. However, there are still some shortcomings of conventional ray-based Kirchhoff migration, such as caustics (Babich and Popov 1989; Popov 1982, 2002) and multipathing problem (Bleistein 1999; Hill 2001; Gray et al. 2002; Liu and Palacharla 2011). Conversely, Gaussian beam migration (GBM) avoids the thorny problem of amplitude singularity in the vicinity of caustics using dynamic ray tracing with a complex-valued initial beam parameter. In the meantime, its decomposition of local plane wave in different directions enables it to propagate different angles to subsurface image point independently, which allows us to deal with multipathing. All of these characteristics make GBM a robust and versatile depth imaging tool, with accuracy comparable to wave equation migration and with flexibility and efficiency comparable to Kirchhoff migration.

GBM has been recognized as a flexible, accurate and elegant depth imaging technique. Hill $(1990,2001)$ proposed the basic poststack and prestack GBM schemes. Briefly, his method can be explained as follows: Hill used a local 
slant-stack approximation with a series of Gaussian window, then ray tracing from the beam center location, finally plane wave components in different directions are back-propagated to the subsurface image point. If a phase correction factor is applied to compensate the phase change of Gaussian beams emanating at geophone locations different from beam center locations, this approximation will be valid when the velocity field near the surface is constant. Unfortunately, it is likely to break down in the condition that surface elevation varies rapidly. For these situations, many researchers extended their work and have made progress. Nowack et al. (2003) and Gray (2005) developed Hill's method to common-shot and common-receiver gathers in order to accommodate the requirements of orthogonal land and marine bottom cable geometries. Meantime, it has been extended to complex topography (Gray 2005; Yue et al. 2010, 2012). In addition, taking into account the complexity of subsurface media, GBM has been applied to elastic media (Casasanta and Gray 2015; Yang et al. 2017) and anisotropic media (Alkhalifah 1995; Zhu et al. 2007; Protasov 2015; Li et al. 2018). For the true amplitude GBM, one branch is proposed by Gray and Bleistein (2009). Another branch is the least squares GBM (Hu et al. 2016; Yang et al. 2018). Moreover, the true amplitude GBM will be benefit to amplitude-versus-angle (AVA) and lithological analysis. A common feature of all these methods is to form plane wave using the local slant stack and then to project it to subsurface according to the traveltime relationship, which can be categorized as traveltime-based GBM (Yang and Zhu 2017).

On the other hand, Popov et al. (2007, 2008, 2010) provided the promising method similar to wave equation migration, which adopts frequency-domain Green's function to construct time domain direct and back-propagated wavefields and then applies crosscorrelation imaging condition. Unlike the traveltime-based GBM, the subsurface image point is determined by the maximum of coherency between the source and receiver wavefields, which is remarkably similar to reverse time migration (RTM). Besides, it carries out the ray tracing from the subsurface image point to the geophone, so it has the capacity to achieve accurate target-oriented imaging. Thereafter, Huang et al. (2017) extended Popov's method (2010) to elastic media; they calculated decoupled back-propagated wavefields by introducing elastic Green's functions of Pand S-waves, which is expressed by a summation of elastodynamic Gaussian beam. Furthermore, considering the viscosity of subsurface media, Bai et al. (2016) proposed a multiple-component Gaussian beam reverse time migration (GBRTM) to implement seismic records compensation for frequency-dependent absorption and dispersion. Likewise, these methods can be categorized as wavefieldbased GBM. Although the wavefield-based GBM can obtain better results than the traditional traveltime-based
GBM, it is difficult to be applied in industry production because of prohibitive computational cost. Overall, in spite of some drawbacks, the wavefield-based GBM still provides us a new idea about accurate target-oriented imaging using Gaussian beam.

For large-scale 3D seismic data, target-oriented reservoir imaging is more attractive than traditional full-volume migration because of high efficiency and short turnaround period (Liu and Wang 2008). For example, in time-lapse reservoir study, efficiently computing local images close to reservoir is important to investigate reservoir dynamic status during oil and gas exploration. Because the quality of the image results is not a linear function of the number of the input shots, the main reflectors can be depicted from a small part of shots; that is, this method enables us to significantly reduce the original datasets in the case when a detailed migrated result is not required. In addition, the image points of target-oriented reservoir imaging are determined point by point, for example, Popov's method (2010). On the contrary, Hill's method (2001) is a data-driven algorithm, which mainly focuses on the data analysis. Firstly, it extracts the sampling point stored in the datasets (local slant-stack), which contains traveltime and attribute characteristics of acquisition such as coordinates of sources and receivers, offset and azimuth, and so on. Then it projects the sampling points to image domain according to traveltime isochron.

It is universally acknowledged that Hill's method is competitive in view of computational efficiency and Popov's method is attractive in term of the accurate targetoriented imaging. It should be noted as well that Popov's method is relatively inefficient on account of the calculation and storage of wavefield at multiple time.

In this paper, we start by describing prestack GBM and analyze the main migration procedure. Combining these methods (Hill 2001; Gray and Bleistein 2009; Popov et al. 2010), we propose a target-oriented GBM using a modified ray tracing scheme in order to satisfy the demands of target-oriented imaging. Next, considering that computation in frequency domain is particularly inefficiency, we have derived a time domain formula using inverse Fourier transform and Hilbert transform. Meantime, we present an extraction strategy of angle domain common image gathers (ADCIGs) using the proposed method to meet the demands of the iterative velocity-model-building and the AVA analysis. Furthermore, we provide a single-shot work flow of subdomain parallel algorithm and implement numerical experimentations on synthetic and field data to confirm the validity and the target-oriented imaging capability of our method. Finally, we make a discussion from the computational grid and the migration velocity analysis, respectively. 


\section{Theory}

Prestack GBM is proposed first for common-offset gathers (Hill 2001; Albertin et al. 2004) and later is extended to common-shot gathers (Nowack et al. 2003; Gray 2005). The above theories of prestack GBM merely emphasize its kinematic imaging capability without considering its amplitude fidelity; hence, Gray and Bleistein (2009) presented true amplitude GBM based on true amplitude wave equation migration (Zhang et al. 2007) and produced ADCIGs. Meantime, prestack GBM is applied to subsurface target-oriented imaging and it carries out the ray tracing from the subsurface image point to the geophone (Popov et al. 2010). In general, prestack GBM is implemented by the crosscorrelation between upgoing and downgoing wavefields, constructed by the Green's functions expressed in Gaussian beam.

\subsection{Review of Gaussian beam migration}

According to Gray and Bleistein (2009), the 2D upgoing and downgoing wavefields expression in frequency domain has the following form

$$
\begin{aligned}
P_{\text {up }}\left(\mathbf{x}_{0} ; \mathbf{x}_{\mathbf{s}} ; \omega\right) & =2 \int \mathrm{d} x_{\mathrm{g}} P_{\text {up }}\left(\mathbf{x}_{\mathbf{g}} ; \mathbf{x}_{\mathbf{s}} ; \omega\right) \frac{\cos \theta_{\mathrm{g}}}{V_{\mathrm{g}}} \sqrt{-i \omega} A_{\mathrm{ART}, \mathrm{g}} \exp \left[-i \omega \tau_{\mathrm{g}}\right] \\
& =-2 \int \mathrm{d} x_{\mathrm{g}} P_{\mathrm{up}}\left(\mathbf{x}_{\mathrm{g}} ; \mathbf{x}_{\mathbf{s}} ; \omega\right) \frac{\cos \theta_{\mathrm{g}}}{V_{\mathrm{g}}} i \omega G^{*}\left(\mathbf{x}_{\mathbf{0}} ; \mathbf{x}_{\mathrm{g}} ; \omega\right),
\end{aligned}
$$

$$
\begin{aligned}
P_{\text {down }}^{*}\left(\mathbf{x}_{\mathbf{0}} ; \mathbf{x}_{\mathrm{s}} ; \omega\right) & =-2 \frac{\cos \theta_{\mathrm{s}}}{V_{\mathrm{s}}} \sqrt{-i \omega A_{\mathrm{ART}, \mathrm{s}} \exp \left[-i \omega \tau_{\mathrm{s}}\right]} \\
& =2 \frac{\cos \theta_{\mathrm{s}}}{V_{\mathrm{s}}} i \omega G^{*}\left(\mathbf{x}_{\mathbf{0}} ; \mathbf{x}_{\mathbf{s}} ; \omega\right)
\end{aligned}
$$

here $P_{\text {up }}$ and $P_{\text {down }}$ are the upgoing (recorded) and the downgoing (source) wavefields, respectively, and $*$ denotes complex conjugation; $V_{\mathrm{s}}$ and $V_{\mathrm{g}}$ are surface velocities at the source $\mathbf{x}_{\mathrm{s}}$ and geophone $\mathbf{x}_{\mathrm{g}} ; \theta_{\mathrm{s}}$ and $\theta_{\mathrm{g}}$ are takeoff angles the rays from source and geophone location to image points $\mathbf{x}_{\mathbf{0}} ; \tau_{\mathrm{s}}, \tau_{\mathrm{g}}$ and $A_{\mathrm{ART}, \mathrm{s}}, A_{\mathrm{ART}, \mathrm{g}}$ are real-valued traveltimes and amplitudes from asymptotic ray theory (ART); and $G\left(\mathbf{x}_{0} ; \mathbf{x} ; \omega\right)$ is the Green's function for the wave equation.

For the Gaussian beam method, in order to obtain the main asymptotic term of the Green's function, we must sum Gaussian beams over angles $\theta$ emanated from the source point $\mathbf{x}$ in different directions. Accordingly, the Green's function expression can be expressed:

$G\left(\mathbf{x}_{\mathbf{0}} ; \mathbf{x} ; \omega\right)=\frac{i}{4 \pi} \int_{0}^{2 \pi} A \exp \left[i \omega \tau(s)+\frac{i \omega}{2} \frac{P(s)}{Q(s)} n^{2}\right] \mathrm{d} \theta$, where variable $s$ is the path length along the ray, $A$ is the amplitude of Gaussian beam and $\tau(s)$ is the traveltime along the raypath, obtained by solving Eq. (4); $P(s)$ and $Q(s)$ that determine the wavefront curvature and the beam width (Hill 1990) are the dynamic parameters obtained by solving Eq. (5)

$\frac{\mathrm{d} x_{i}(s)}{\mathrm{d} \tau}=v^{2}(s) p_{i}(s), \frac{\mathrm{d} p_{i}(s)}{\mathrm{d} \tau}=-\frac{1}{v(s)} \frac{\partial v(s)}{\partial x_{i}}$,

$\frac{\mathrm{d} Q(s)}{\mathrm{d} \tau}=v^{2}(s) P(s), \frac{\mathrm{d} P(s)}{\mathrm{d} \tau}=-\frac{1}{v(s)} \frac{\partial^{2} v(s)}{\partial n^{2}} Q(s)$,

$\frac{\partial^{2} v(s)}{\partial n^{2}}=\frac{\partial^{2} v(s)}{\partial x^{2}} \cos ^{2} \theta-2 \frac{\partial^{2} v(s)}{\partial x \partial z} \cos \theta \sin \theta+\frac{\partial^{2} v(s)}{\partial z^{2}} \sin ^{2} \theta$,

here $x_{i}(s)$ are the ray coordinates in the Cartesian coordinates; $s$ and $n$ are ray-centered coordinates; $p_{i}(s)$ are the components of the slowness vector along the raypath.

Assume that $A_{\mathrm{s}}, A_{\mathrm{g}}$ and $T_{\mathrm{s}}, T_{\mathrm{g}}$ are the complex amplitudes and traveltimes at the source $\mathbf{x}_{\mathrm{s}}$ and geophone $\mathbf{x}_{\mathrm{g}}$, respectively. Thus Eq. (3) can be simplified to the following form

$$
\begin{aligned}
& G\left(\mathbf{x}_{\mathbf{0}} ; \mathbf{x}_{\mathbf{s}} ; \omega\right)=\frac{i}{4 \pi} \int_{0}^{2 \pi} \mathrm{d} \theta_{\mathrm{s}} A_{\mathrm{s}} \exp \left[i \omega T_{\mathrm{s}}\right], \\
& G\left(\mathbf{x}_{\mathbf{0}} ; \mathbf{x}_{\mathbf{g}} ; \omega\right)=\frac{i}{4 \pi} \int_{0}^{2 \pi} \mathrm{d} \theta_{\mathrm{g}} A_{\mathrm{g}} \exp \left[i \omega T_{\mathrm{g}}\right],
\end{aligned}
$$

the above Green's functions can avoid caustics and accommodate multipathing only with considerable effort, which is different from the Green's function in Kirchhoff migration. Next, we denote that

$$
\begin{aligned}
A_{\mathrm{s}}(\omega) & =\operatorname{Re}\left[A_{\mathrm{s}}(|\omega|)\right]+i \operatorname{sgn}(\omega) \operatorname{Im}\left[A_{\mathrm{s}}(|\omega|)\right], \\
A_{\mathrm{g}}(\omega) & =\operatorname{Re}\left[A_{\mathrm{g}}(|\omega|)\right]+i \operatorname{sgn}(\omega) \operatorname{Im}\left[A_{\mathrm{g}}(|\omega|)\right],
\end{aligned}
$$

Equation (7) means that the complex amplitude at negative frequency is the complex conjugate of complex amplitude at positive frequency. Furthermore, considering that the complex conjugate of $e$ exponent equal to the complex conjugate of its power exponent, we can obtain the complex conjugate of Green's function as follows:

$$
\begin{aligned}
G^{*}\left(\mathbf{x}_{\mathbf{0}} ; \mathbf{x}_{\mathrm{s}} ; \omega\right) & =\frac{i}{4 \pi} \int_{0}^{2 \pi} \mathrm{d} \theta_{\mathrm{s}} A_{\mathrm{s}}^{*} \exp \left[\left(i \omega T_{\mathrm{s}}\right)^{*}\right]=\frac{i}{4 \pi} \int_{0}^{2 \pi} \mathrm{d} \theta_{\mathrm{s}} A_{\mathrm{s}}^{*} \exp \left[-i \omega T_{\mathrm{s}}^{*}\right], \\
G^{*}\left(\mathbf{x}_{\mathbf{0}} ; \mathbf{x}_{\mathbf{g}} ; \omega\right) & =\frac{i}{4 \pi} \int_{0}^{2 \pi} \mathrm{d} \theta_{\mathrm{g}} A_{\mathrm{g}}^{*} \exp \left[\left(i \omega T_{\mathrm{g}}\right)^{*}\right]=\frac{i}{4 \pi} \int_{0}^{2 \pi} \mathrm{d} \theta_{\mathrm{g}} A_{\mathrm{g}}^{*} \exp \left[-i \omega T_{\mathrm{g}}^{*}\right] .
\end{aligned}
$$


Zhang et al. (2007) proposes the following crosscorrelation imaging condition in frequency domain:

$I\left(\mathbf{x}_{\mathbf{0}} ; \mathbf{x}_{\mathbf{s}}\right)=-i \int \mathrm{d} \omega \operatorname{sgn}(\omega) P_{\text {up }}\left(\mathbf{x}_{\mathbf{0}} ; \mathbf{x}_{\mathrm{s}} ; \omega\right) P_{\text {down }}^{*}\left(\mathbf{x}_{\mathbf{0}} ; \mathbf{x}_{\mathbf{s}} ; \omega\right)$,

and inserting Eqs. (1) and (2) into Eq. (9), we obtain

$$
\begin{aligned}
I\left(\mathbf{x}_{\mathbf{0}} ; \mathbf{x}_{\mathbf{s}}\right)=- & 4 \int \mathrm{d} \omega i \omega|\omega| \frac{\cos \theta_{\mathrm{s}}}{V_{\mathrm{s}}} G^{*}\left(\mathbf{x}_{\mathbf{0}} ; \mathbf{x}_{\mathrm{s}} ; \omega\right) \\
& \times \int \mathrm{d} x_{\mathrm{g}} P_{\mathrm{up}}\left(\mathbf{x}_{\mathrm{g}} ; \mathbf{x}_{\mathbf{s}} ; \omega\right) \frac{\cos \theta_{\mathrm{g}}}{V_{\mathrm{g}}} G^{*}\left(\mathbf{x}_{\mathbf{0}} ; \mathbf{x}_{\mathrm{g}} ; \omega\right) .
\end{aligned}
$$

Although our method differs from Hill's (2001) and (Gray and Bleistein 2009) derivation of prestack GBM, most of the steps are identical. Usually, we need to first determine a series of beam center locations and then adopt the local slant-stack strategy because the Gaussian beam wavefront curvature is zero at its initial location on the beam center. In addition, the local slant-stack results in some kinematic error because the wavefront curvature gradually becomes nonzero when away from the beam center, even then, this error usually is negligible. It should be noted as well that it is likely to break down in the condition that the receiver location is far from the beam center and surface velocity and/or surface elevation vary rapidly. For these situations, on the one hand, Hill (1990) introduced a Gaussian window function to ensure that the traces far from a beam center are downweighted exponentially relative to traces close to a beam center, so the amplitude error is slight. On the other hand, Gray (2005) extended Hill's method to complex topography by a modification of local slant-stack. Instead of these choices, we insert Eq. (8) into Eq. (10) directly without the slantstack approximation and rearrange terms to arrive at the migration formula in frequency domain:

$$
\begin{aligned}
I\left(\mathbf{x}_{\mathbf{0}} ; \mathbf{x}_{\mathrm{s}}\right)= & \frac{1}{4 \pi^{2}} \int \mathrm{d} x_{\mathrm{g}} \int \mathrm{d} \omega i \omega \frac{\cos \theta_{\mathrm{s}}}{V_{\mathrm{s}}} \int_{0}^{2 \pi} \mathrm{d} \theta_{\mathrm{s}} A_{\mathrm{s}}^{*} \exp \left[-i \omega T_{\mathrm{s}}^{*}\right] \\
& \times \frac{\cos \theta_{\mathrm{g}}}{V_{\mathrm{g}}} \int_{0}^{2 \pi} \mathrm{d} \theta_{\mathrm{g}} A_{\mathrm{g}}^{*} \exp \left[-i \omega T_{\mathrm{g}}^{*}\right] W\left(\mathbf{x}_{\mathrm{g}} ; \mathbf{x}_{\mathrm{s}} ; \omega\right),
\end{aligned}
$$

where

$W\left(\mathbf{x}_{\mathbf{g}} ; \mathbf{x}_{\mathbf{s}} ; \omega\right)=|\omega| P_{\text {up }}\left(\mathbf{x}_{\mathbf{g}} ; \mathbf{x}_{\mathbf{s}} ; \omega\right)$,

and it represents frequency weighted seismic records. Equation (11) is a quadruple integral of the geophone location, frequency, takeoff angle emanated from the source and receiver, which expresses a migrated result as a sum over all receivers locations, where each geophone contributes partial images in different directions.

It is obvious that straightforward implementation of Eq. (11) would demand numerous costly summation over frequency $\omega$. To reduce the computational load of Eq. (11), we exchange the order of integration and obtain

$$
\begin{aligned}
I\left(\mathbf{x}_{\mathbf{0}} ; \mathbf{x}_{\mathbf{s}}\right)= & \frac{1}{4 \pi^{2}} \int \mathrm{d} x_{\mathrm{g}} \int_{0}^{2 \pi} \mathrm{d} \theta_{\mathrm{s}} \int_{0}^{2 \pi} \mathrm{d} \theta_{\mathrm{g}} \int i \omega \mathrm{d} \omega \frac{\cos \theta_{\mathrm{s}}}{V_{\mathrm{s}}} \frac{\cos \theta_{\mathrm{g}}}{V_{\mathrm{g}}} \\
& \times A_{\mathrm{s}}^{*} \exp \left[-i \omega T_{\mathrm{s}}^{*}\right] A_{\mathrm{g}}^{*} \exp \left[-i \omega T_{\mathrm{g}}^{*}\right] W\left(\mathbf{x}_{\mathrm{g}} ; \mathbf{x}_{\mathrm{s}} ; \omega\right),
\end{aligned}
$$

here $\cos \theta_{\mathrm{s}} / V_{\mathrm{s}}$ and $\cos \theta_{\mathrm{g}} / V_{\mathrm{g}}$ are the frequency-independent variables and $\int \mathrm{d} \theta_{\mathrm{s}} \int \mathrm{d} \theta_{\mathrm{g}}$ means the integration for each beam. Thus, the innermost integration can be understood as the crosscorrelation at an image point between the beams emanated from the source and receiver. Meantime, considering traveltime and amplitude are complex, the integrand is a complex as well. We denote

$$
\begin{gathered}
T_{\mathrm{re}}=\operatorname{Re}\left(T_{\mathrm{s}}^{*}+T_{\mathrm{g}}^{*}\right), A_{\mathrm{re}}=\operatorname{Re}\left(A_{\mathrm{s}}^{*} A_{\mathrm{g}}^{*}\right) \\
T_{\mathrm{im}}=\operatorname{Im}\left(T_{\mathrm{s}}^{*}+T_{\mathrm{g}}^{*}\right), A_{\mathrm{im}}=\operatorname{Im}\left(A_{\mathrm{s}}^{*} A_{\mathrm{g}}^{*}\right),
\end{gathered}
$$

then focused on the real part of the integrand, and the quadruple integral can be reduced to triple integral by transforming the integral variable frequency to time using the inverse Fourier transform and Hilbert transform (Gao et al. 2015). Therefore, the migration formula in time domain can be expressed as follows

$$
\begin{aligned}
I\left(\mathbf{x}_{\mathbf{0}} ; \mathbf{x}_{\mathrm{s}}\right)= & \frac{1}{2 \pi} \int \mathrm{d} x_{\mathrm{g}} \int_{0}^{2 \pi} \mathrm{d} \theta_{\mathrm{s}} \int_{0}^{2 \pi} \mathrm{d} \theta_{\mathrm{g}} \frac{\cos \theta_{\mathrm{s}}}{V_{\mathrm{s}}} \frac{\cos \theta_{\mathrm{g}}}{V_{\mathrm{g}}} \\
& {\left[A_{\mathrm{re}} \bar{W}\left(\mathbf{x}_{\mathrm{g}} ; \mathbf{x}_{\mathrm{s}} ; T_{\mathrm{re}}, T_{\mathrm{im}}\right)-A_{\mathrm{im}} \bar{W}_{\mathrm{H}}\left(\mathbf{x}_{\mathbf{g}} ; \mathbf{x}_{\mathrm{s}} ; T_{\mathrm{re}}, T_{\mathrm{im}}\right)\right], }
\end{aligned}
$$

here $\bar{W}$ denotes the inverse Fourier transform of $W$ and subscript $\mathrm{H}$ denotes the Hilbert transform. The detailed derivation process of Eq. (15) is given in Appendix A of this paper. It should be noted as well that the imaginary part of the traveltime, required by Eq. (15) in beam formation before imaging, is determined by ray tracing during migration procedure. In order to avoid repeated calculation, a feasible method is to first calculate the counterpart of $\bar{W}$ and $\bar{W}_{\mathrm{H}}$ and then match the imaginary traveltime of a subsurface image point with the counterpart (Hale 1992b).

\subsection{Target-oriented Gaussian beam migration using a modified ray tracing scheme}

The conventional GBM requires a local slant-stack with a series of Gaussian windows, then ray tracing from the beam 
center location, finally plane waves components in different directions are back-propagated to the underground image point by Green's function. The Green's function is the wavefield generated by a point (impulse or delta function) source. In acoustic media, the Green's function is the pressure generated by an impulse source, which is a scalar. For elastic seismology, the Green's function is the displacement generated by a single force, which is a second-order tensor. Next, we assume that the Green's function $G\left(\mathbf{x}_{\mathbf{0}} ; \mathbf{x}_{\mathbf{g}} ; \omega\right)$ is the response at point $\mathbf{x}_{\mathbf{0}}$ to a source at point $\mathbf{x}_{\mathbf{g}}$. More specifically, if $G\left(\mathbf{x}_{\mathbf{0}} ; \mathbf{x}_{\mathbf{g}} ; \omega\right)$ is the retarded solution, which represents the waves expanding from the point source, then its complex conjugate $G^{*}\left(\mathbf{x}_{\mathbf{0}} ; \mathbf{x}_{\mathbf{g}} ; \omega\right)$ is the advanced solution, which represents waves imploding toward the point source (Hill 2001). According to the reciprocity theorem between the fields and the source, the following relation is established:

$G^{*}\left(\mathbf{x}_{\mathbf{0}} ; \mathbf{x}_{\mathbf{g}} ; \omega\right)=G^{*}\left(\mathbf{x}_{\mathbf{g}} ; \mathbf{x}_{\mathbf{0}} ; \omega\right)$,

where $G^{*}\left(\mathbf{x}_{\mathbf{g}} ; \mathbf{x}_{\mathbf{0}} ; \omega\right)$ is constructed by the rays from the image point $\mathbf{x}_{\mathbf{0}}$ to the geophone location $\mathbf{x}_{\mathbf{g}}$, which is opposite to $G^{*}\left(\mathbf{x}_{\mathbf{0}} ; \mathbf{x}_{\mathbf{g}} ; \omega\right)$. It is obvious that Green's function $G^{*}\left(\mathbf{x}_{\mathbf{g}} ; \mathbf{x}_{\mathbf{0}} ; \omega\right)$ is the appropriate choice in the target-oriented imaging. Simultaneously, assuming that the whole region is $\Omega$ and the target region is $\Omega_{i}$, we derive the target-oriented Gaussian beam migration (TOGBM) formula as follows:

$$
\begin{aligned}
\left.I\left(\mathbf{x}_{\mathbf{0}} ; \mathbf{x}_{\mathrm{s}}\right)\right|_{\mathrm{x}_{\mathrm{v}} \in \Omega_{i}}= & \frac{1}{2 \pi} \int \mathrm{d} x_{\mathrm{g}} \int_{0}^{2 \pi} \mathrm{d} \theta_{\mathrm{s}} \int_{0}^{2 \pi} \mathrm{d} \theta_{\mathrm{g}} \frac{\cos \theta_{\mathrm{s}}}{V_{\mathrm{s}}} \frac{\cos \theta_{\mathrm{g}}}{V_{\mathrm{g}}} \\
& {\left.\left[A_{\mathrm{re}} \bar{W}\left(\mathbf{x}_{\mathbf{g}} ; \mathbf{x}_{\mathrm{s}} ; T_{\mathrm{re}}, T_{\mathrm{im}}\right)-A_{\mathrm{im}} \bar{W}_{\mathrm{H}}\left(\mathbf{x}_{\mathrm{g}} ; \mathbf{x}_{\mathrm{s}} ; T_{\mathrm{re}}, T_{\mathrm{im}}\right)\right]\right|_{\mathbf{x}_{0} \in \Omega_{i}} . }
\end{aligned}
$$

\subsection{Extraction of ADCIGs}

In the traditional sense, crosscorrelation imaging condition is not amplitude-preserved, which will be influenced by the intensity of illumination, for example, the amplitude will be weak in areas of inadequate illumination. However, $\mathrm{Xu}$ et al. (2001) and Zhang et al. (2007) proved that if the image results based on crosscorrelation imaging condition is transformed into angle domain, ADCIGs will contain the angle-dependent reflection coefficient, i.e., crosscorrelation imaging condition is amplitude-preserved in angle domain. Furthermore, ADCIGs can be applied to the iterative velocity-model-building and AVA analysis, so we develop the extraction method for TOGBM.

Extraction of ADCIGs in wave equation migration is mainly based on the space-shift imaging condition and the time shift imaging condition (Save and Fomel 2003, 2005, 2006). It is necessary to first extract local offset domain common image gathers (L-ODCIGs) and time shift common image gathers (TSCIGs), which represent the focusability of wavefields in space direction and in time direction, respectively. Then L-ODCIGs and TSCIGs can be transformed into ADCIGs according to the angular mapping relation. Unlike wave equation migration, ADCIGs in GBM can be directly extracted without mapping transformations because the Gaussian beam contains the subsurface angle information during migration procedure, which is extremely handy and efficient.

According to Gray and Bleistein (2009), the similar extraction steps of ADCIGs for TOGBM can be given as follows: (1) summing the ray angles from the source and image point to calculate the opening angle for migrating a sample onto an image location; (2) placing the migrated amplitude into the position of ADCIGs according to that opening angle. The detailed formula and derivation process on how to extract ADCIGs for TOGBM are discussed in Appendix B of this paper.

\section{TOGBM workflow}

The work flow of the conventional GBM can be briefly summarized in four steps: (1) determining the beam center location according to the beam center interval; (2) local slantstack with a series of Gaussian windows; (3) ray tracing from the beam center location; (4) plane waves components in different directions are back-propagated to the subsurface image point. However, as shown in Fig. 1, our method adopts a upgoing ray tracing scheme, which requires the rays from the subsurface image point to the geophone in different takeoff angles $\alpha_{\mathrm{g}}$; it is possible for us to select one point in migration domain randomly, i.e., the migration process of each image point is absolutely independent; thus, our method can achieve the target-oriented imaging which is of significant importance for 3D migration with huge seismic data.

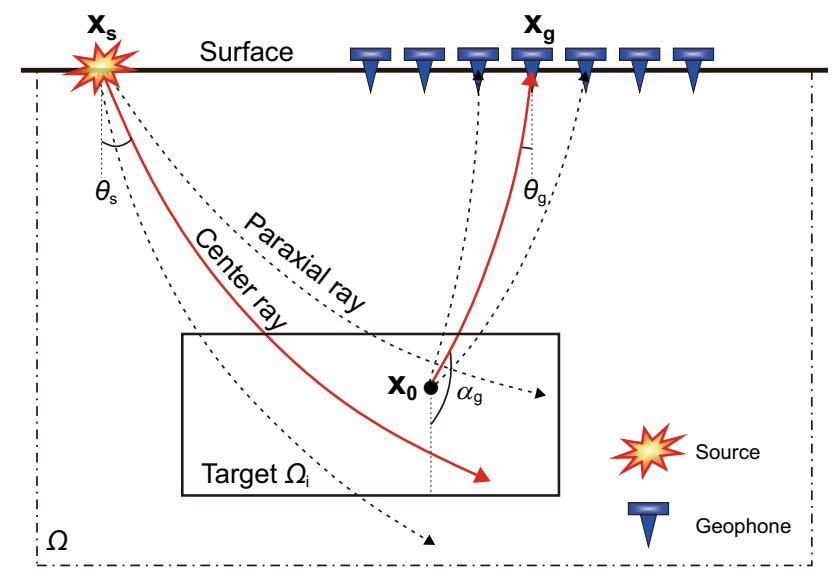

Fig. 1 Diagram of the target-oriented Gaussian beam migration 
Popov et al. (2010) propose a high level of algorithm parallelization. Similarly, according to Eq. (17), we design the following single-shot work flow (see Fig. 2) to implement the algorithm. We first take the velocity field and single-shot seismic records as the inputs. Then before migration of each target region, we carry out the seismic records processing using Fourier transform and Hilbert transform. Next, we make full use of the characteristic that the migration

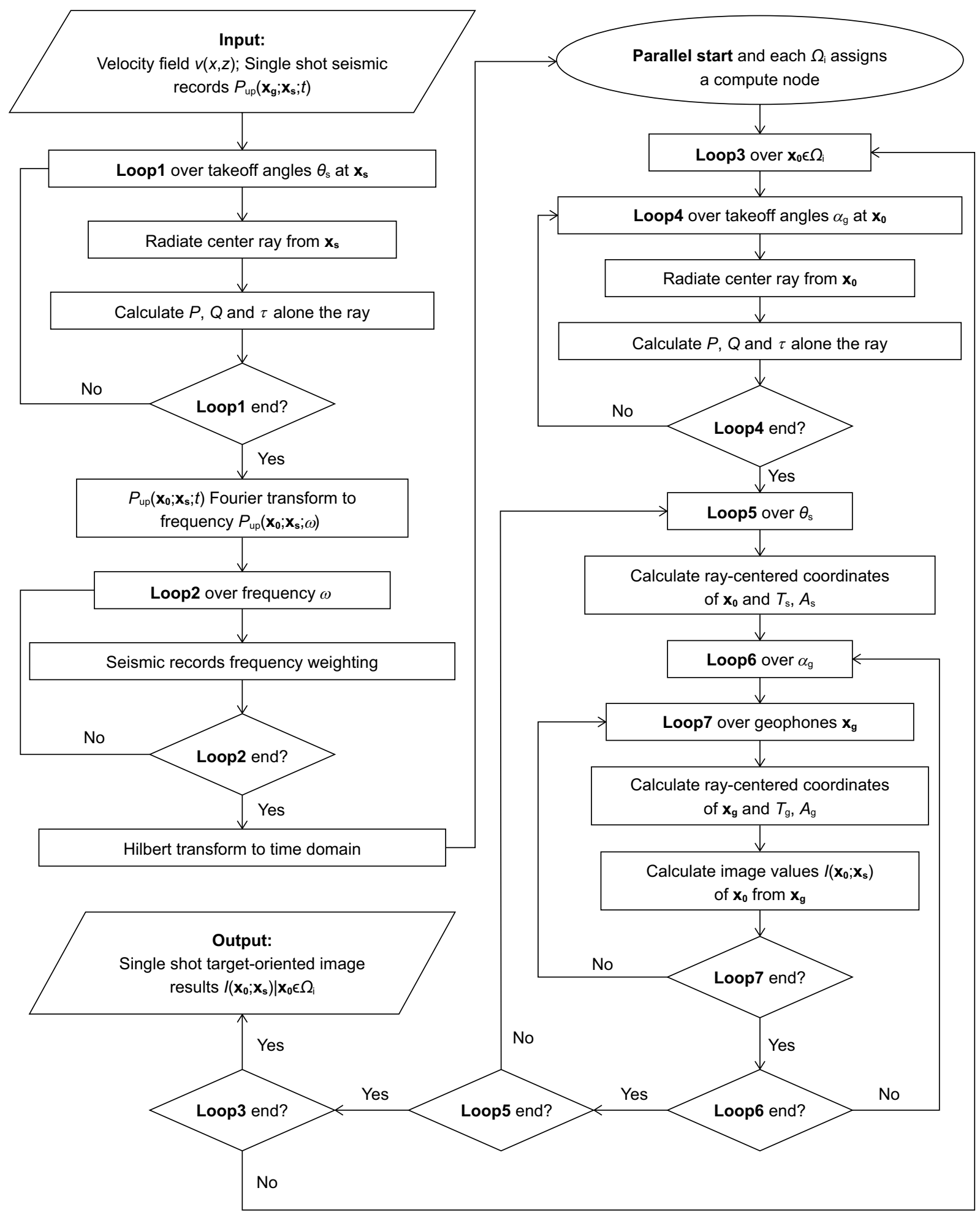

Fig. 2 Flowchart of TOGBM for single-shot seismic records 
process of each image point is absolutely independent and adopt a parallel algorithm that each target region assigns a compute node. It is obvious that the parallel algorithm of multiple regions can enhance the computational efficiency significantly. Finally, we output single target-oriented image results.

\section{Numerical examples}

In this section, based on the above theory and algorithm, we test the performance of our method using five numerical examples including four synthetic examples and a field data example. The first example is the 2D SEG/EAGE salt model as shown in Fig. 3. The model is characterized by the high velocity of the salt body, which is about twice as fast as the sedimentary layer, the sharp changes in lateral velocity, and very large dip angle of the left flank. The grid size of the model is $645 \times 300$, and sample intervals of distance and depth are $24 \mathrm{~m}$ and $12 \mathrm{~m}$, respectively. Dataset has 325 shots

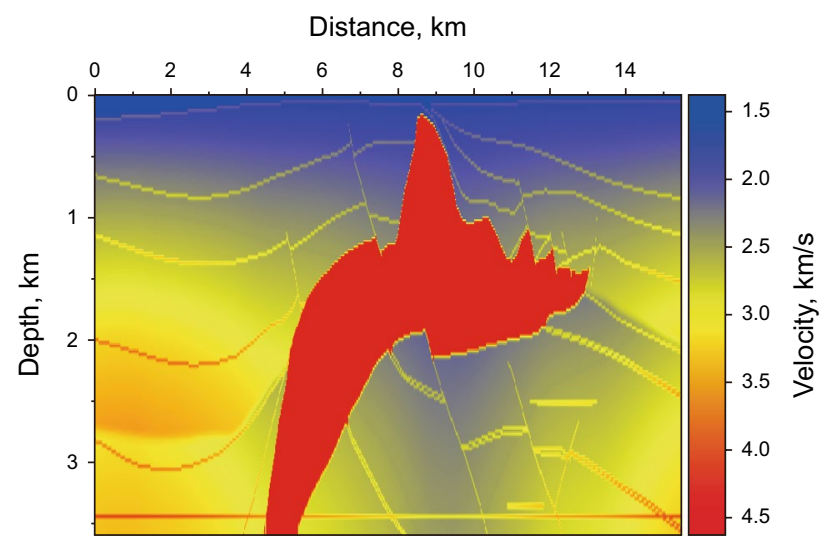

Fig. 3 The velocity of the 2D SEG/EAGE salt model

(a)

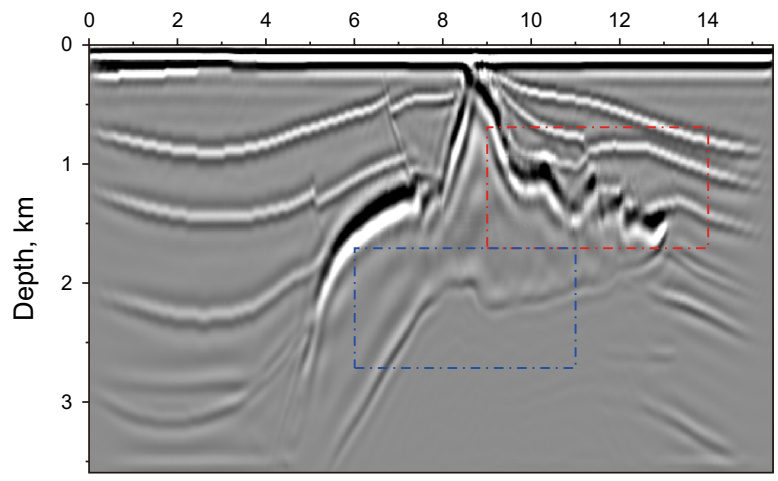

and 88 geophones with $24-\mathrm{m}$ receiver interval per shot, and each trace has 626 samples with sample rate $8 \mathrm{~ms}$.

Figure 4 shows the depth images migrated with the conventional method and the proposed method. It is obvious that the conventional method (see Fig. 4a) restores accurately the basic structure of the model, but it produces the poor image results at flanks and bottoms of the salt. Compared to the conventional method, our method generates the clearer image profiles for the adumbration of the salt. Two magnified rectangular windows from these two migration results are displayed in Fig. 5. Apparently, the event is more continuous migrated by our method (see Fig. $5 b$, d) than the conventional method (see Fig. 5a, c). It should be noted as well that the reducing of frequency also inevitably appears in our method, which is likely to result from (1) back-propagation of the seismic records without the local slant-stack approximation and (2) the construction of Green's function with upgoing ray tracing strategy.

The second example is implemented on an ocean model to demonstrate the target-oriented imaging capability of our method. The dataset is simulated by a staggered-grid finitedifference method with tenth-order accuracy in space and second-order accuracy in time, and the dominant frequency is $30 \mathrm{~Hz}$. As shown in Fig. 6, the model contains two steep faults, and high-velocity natural gas hydrate reservoirs are formed on both sides of the fault. Besides, there is a lowvelocity oil and gas reservoir in the left sag, and the bottom of the model is the intrusive rock mass with steep dip angle. The ocean model is $8.0 \mathrm{~km}$ long and $4.0 \mathrm{~km}$ deep. Beginning at $1.0 \mathrm{~km}$ from the left edge of this model, a total of 61 shots are simulated with a shot interval of $100 \mathrm{~m}$, and 401 geophones are evenly distributed on the surface with $20-\mathrm{m}$ interval. The record duration is $3 \mathrm{~s}$, and time sample interval is $4 \mathrm{~ms}$.

Likewise, we first obtain the migrated result by the conventional method (see Fig. 7). Although the main structures can be basically identified, there are some noise near the

(b)

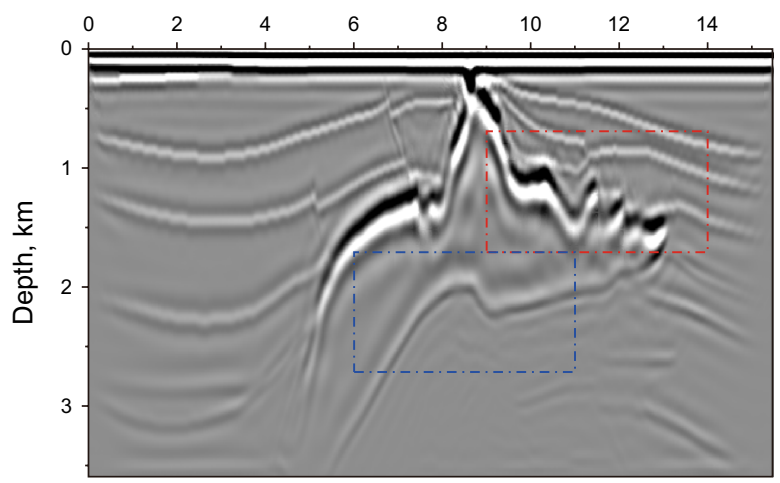

Fig. 4 The GBM results of the 2D SEG/EAGE salt model using: a the conventional method and, $\mathbf{b}$ our method 

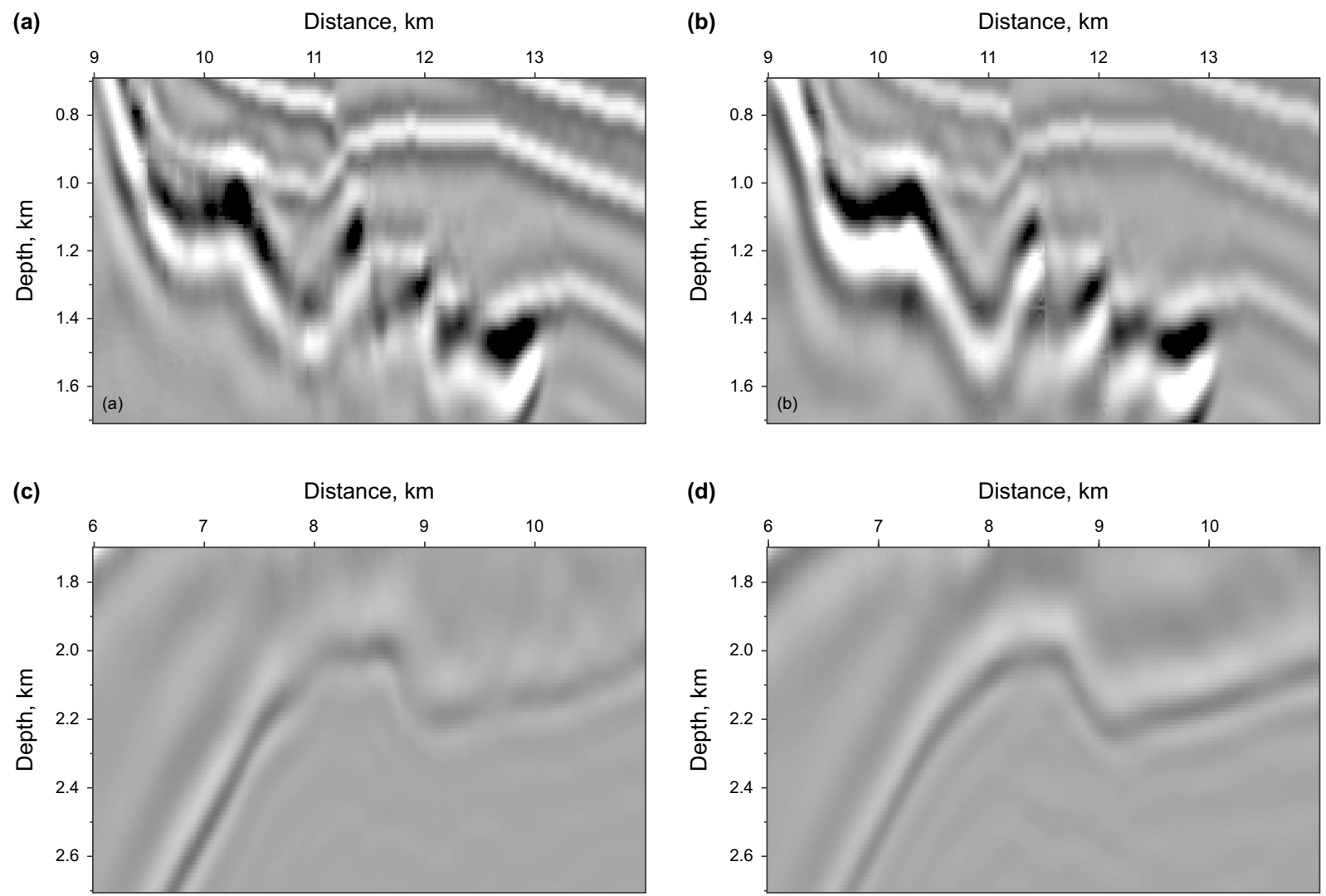

Fig. 5 Magnified views of migration results in Fig. 4. The magnified zones are from $(9.0 \mathrm{~km}, 0.7 \mathrm{~km})$ to $(14 \mathrm{~km}, 1.7 \mathrm{~km})$ and $(6.0 \mathrm{~km}, 1.7 \mathrm{~km})$ to $(11 \mathrm{~km}, 2.7 \mathrm{~km})$, as the red rectangular window and the blue rectangular window in Fig. 4. For the red frame, panels $\mathbf{a}$ and $\mathbf{b}$ are the magnified views of migration results migrated by the conventional method and our method, respectively. Planes $\mathbf{c}$ and $\mathbf{d}$ are the magnified views for the blue rectangular windows

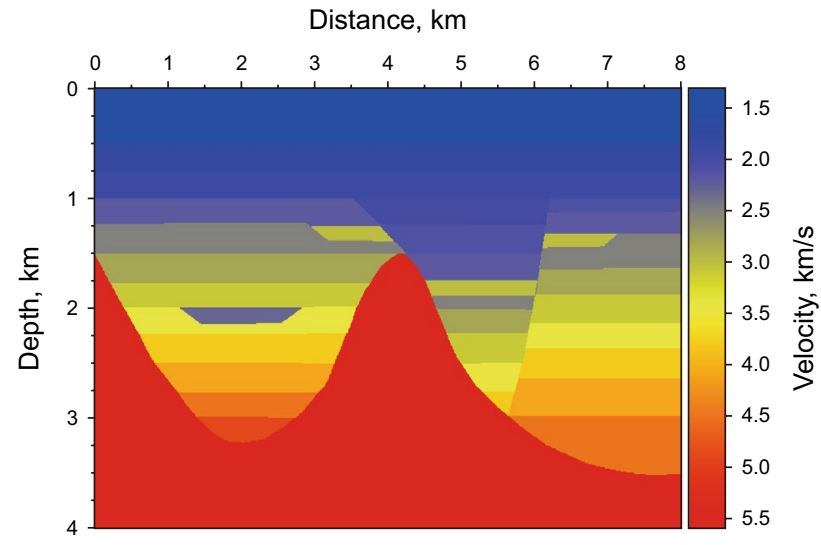

Fig. 6 The velocity of the ocean model

faults. Considering the characteristic of the ocean model, we select four target regions (see Fig. 9) to migrate simultaneously using different computer nodes. Before the migration, we need to obtain a smoothed velocity model (see Fig. 8) with a damping least squares method because ray tracing requires the second-order derivations of the velocity,

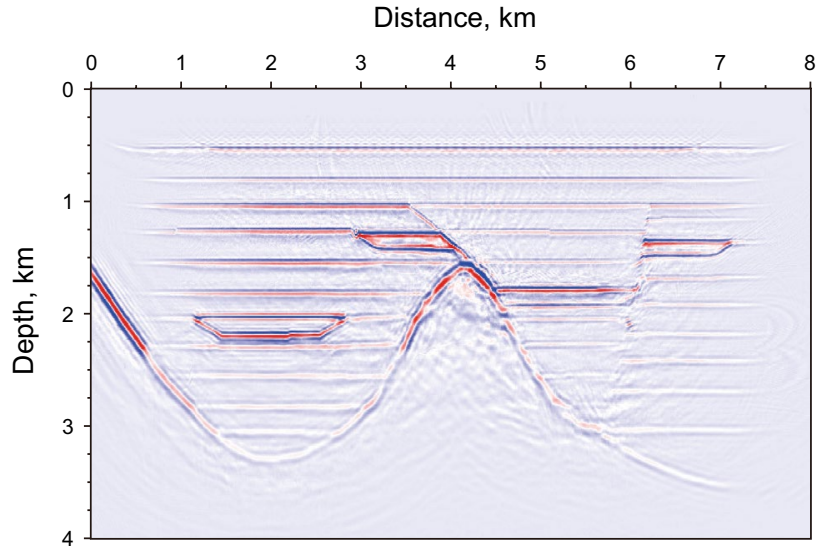

Fig. 7 The GBM result of the ocean model using the conventional method

which ensures the stability of dynamic ray tracing (Popov et al. 2010). Besides, we draw the 1st and 41st shot raypath (marked by white lines in Fig. 8) and two image points in target regions (marked by red lines in Fig. 8). Figure 9 shows 


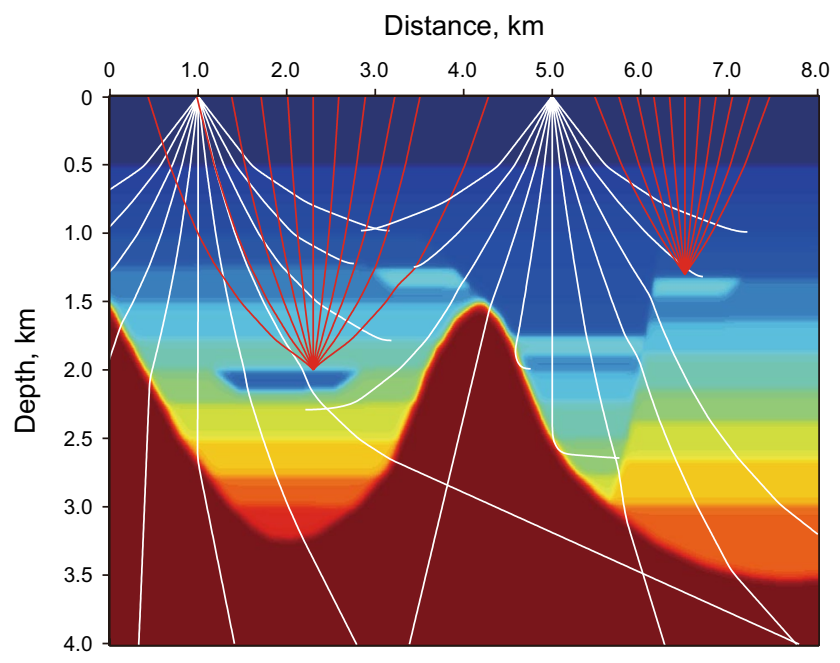

Fig. 8 The smoothed velocity of the ocean model and raypaths from the sources (marked by white lines) and the image points (marked by red lines)

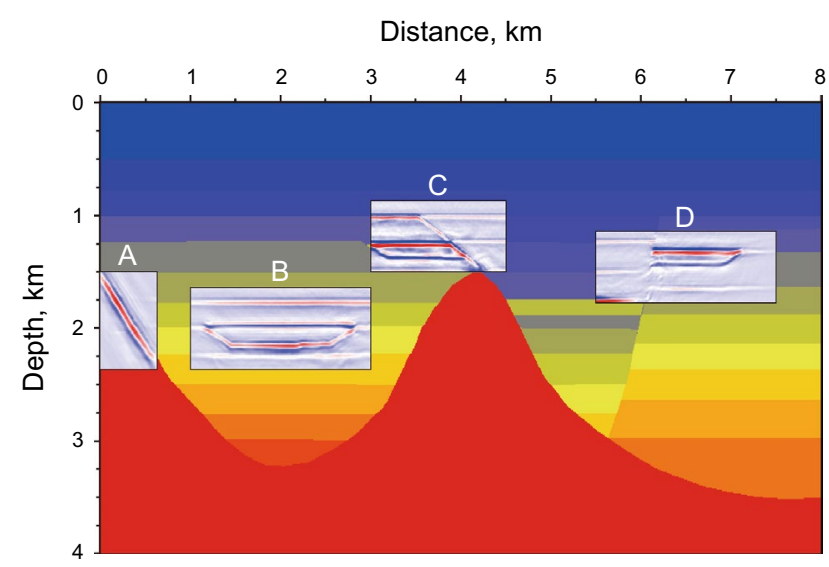

Fig. 9 The target-oriented imaging results of the ocean model at four target regions. Region A, B, C and D contain salt dome basement, low-velocity body, faults and high-velocity body, respectively

the image results in four target regions, and it is easy to see that our method produces a clear image and image position is consistent with model. Furthermore, we compare the results in four target regions by the conventional method and our method. As shown in Fig. 10, our method makes less noise near the faults and produces a clearer profile than the convention method; only dispersion phenomenon still appears owing to the input synthetic seismic records using the finite-difference modeling method. Furthermore, we compare the efficiency of GBM and TOGBM for the ocean model, and the results are descripted in Table 1. As expected, the cost of TOGBM is about eight times of that of GBM for full model, because upgoing rays need to be shot from every image location in the target region that is very time-consuming. However, TOGBM enhances the computation efficiency significantly compared to GBM for the small target region, especially for target region A of the ocean model.

In the third example, we use the BP velocity model (Billette and Brandsberg-Dahl 2004) to test the adaptability of our method for large subsurface volumes. As shown in Fig. 11, the distance and depth of the BP model are about $67 \mathrm{~km}$ and $12 \mathrm{~km}$, which are eight times and triple the ocean model (see Fig. 6), respectively. The grid size of the model is $5395 \times 1911$, and sample intervals of distance and depth are $12.5 \mathrm{~m}$ and $6.25 \mathrm{~m}$. The synthetic seismic data consists of 1348 shots and 1201 geophones with $12.5 \mathrm{~m}$ receiver interval per shot, and each trace has 2001 samples with sample rate of $6 \mathrm{~ms}$. For each shot, the offsets range from $-15,000$ to $0 \mathrm{~m}$ and shot increment is $50 \mathrm{~m}$. The size of the complete dataset, divided into 7 pieces over the shot axis, is about $12.43 \mathrm{~GB}$.

It should be noted that BP dataset includes variable density, but we have to equate the density to one throughout the model because GBM is currently based on the classical wave equation without considering density information. We first carry out the conventional method using the BP dataset. The migration result is depicted in Fig. 12. It is obvious that the shallow layers have better image results than the deep layers and the outline of salt body can been recognized. Besides, some small low- and high-speed bodies can arise in the profile as well. Unfortunately, the structure beneath the salt body is almost invisible, which is likely to result from the insufficient density of ray beneath the salt. Furthermore, in order to illustrate the problem of the conventional method, we emanate a fan of rays from all shot locations on the surface and compute the ray folds of subsurface image points according to the coordinates of raypath points. As shown in Fig. 13, the energy of migration result is nearly consistent with the density of ray, i.e., when the ray folds of some region are little, the energy of the events is also weak, for example, the complex rugose multivalued salt body in the left and the deeply rooted salt body in the central part.

For comparison, we select six target regions, the same as (Popov et al. 2010), which is aimed to reconstruct the structure of this complex model containing a complex rugose multivalued salt body in the left part and a deeply rooted salt body in the central part. The target-oriented imaging results are depicted in Fig. 14. The position 

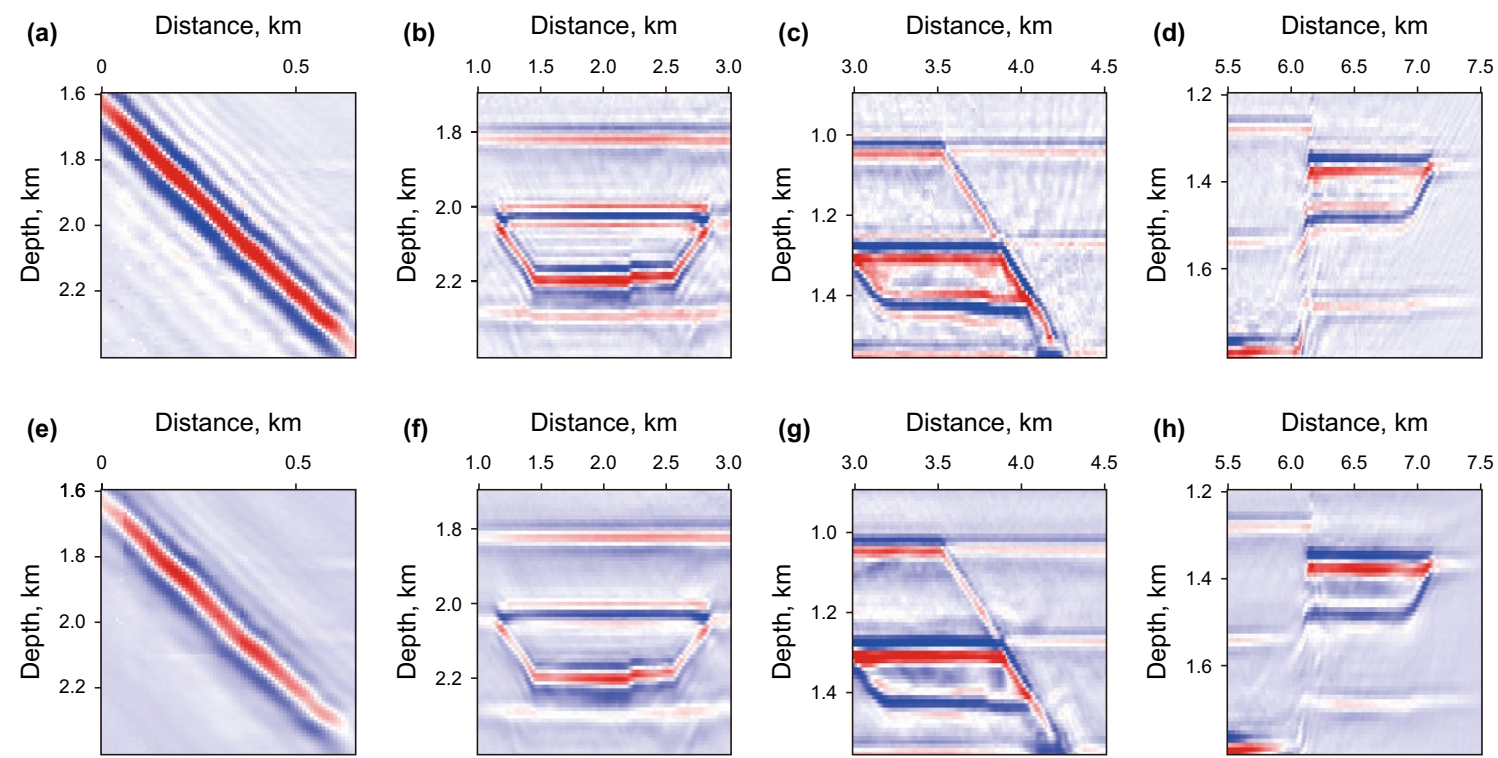

Fig. 10 The comparison of migration results in four target regions. Panels a-d are the close-up views of the migration result (see Fig. 7) using the convention method. Panels $\mathbf{e}-\mathbf{h}$ are the target-oriented imaging results in Fig. 9

Table 1 The comparison of computational costs between GBM and TOGBM for the ocean model. All methods are run on an Intel workstation (Xeon E5-2630 2.2 GHz)

\begin{tabular}{lllllll}
\hline Methods & GBM & TOGBM & & & \\
\cline { 3 - 6 } Target region & All & All & A & B & C & $151 \times 66$ \\
\hline Grid parameters & $801 \times 401$ & $801 \times 401$ & $66 \times 81$ & $201 \times 71$ & $195 \times 61$ \\
Calculating time, min & 176.0 & 1367.5 & 21.3 & 110.9 & 116.2 & 95.7 \\
Normalized time & 1.00 & 7.77 & 0.12 & 0.63 & 0.66 & 0.54 \\
\hline
\end{tabular}

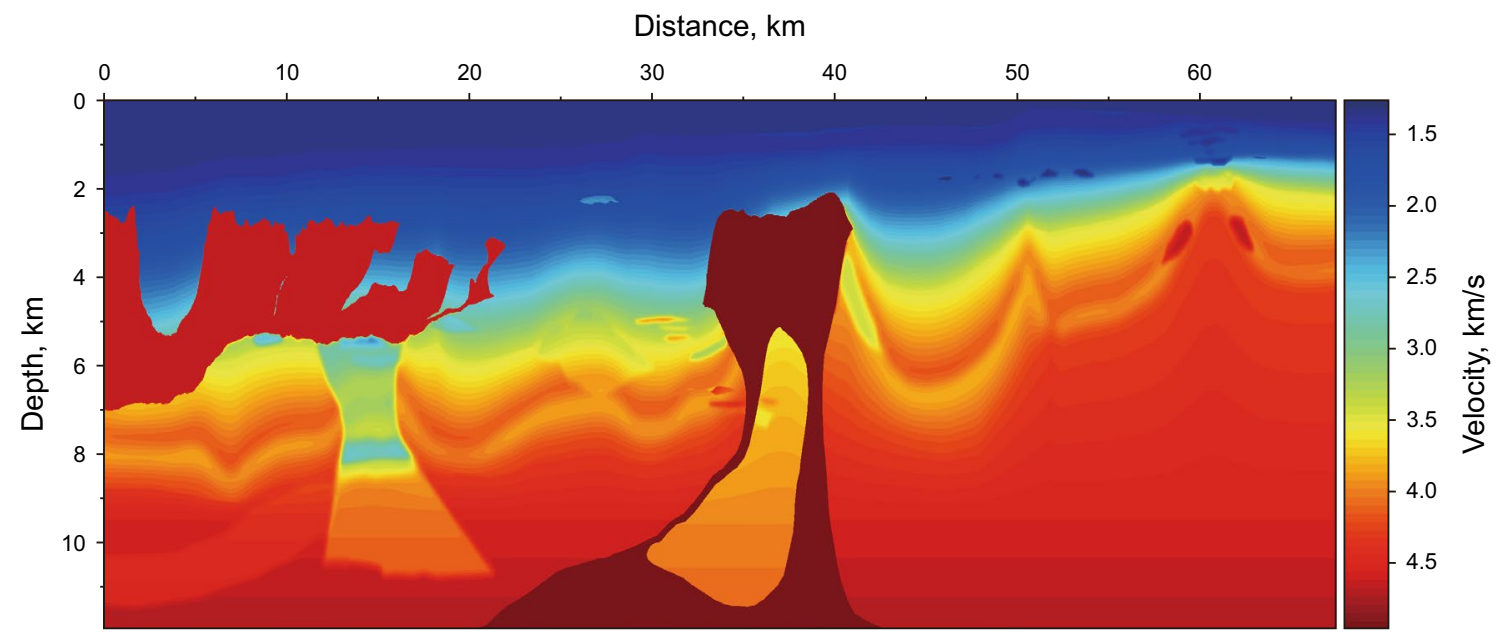

Fig. 11 The velocity of the BP model 


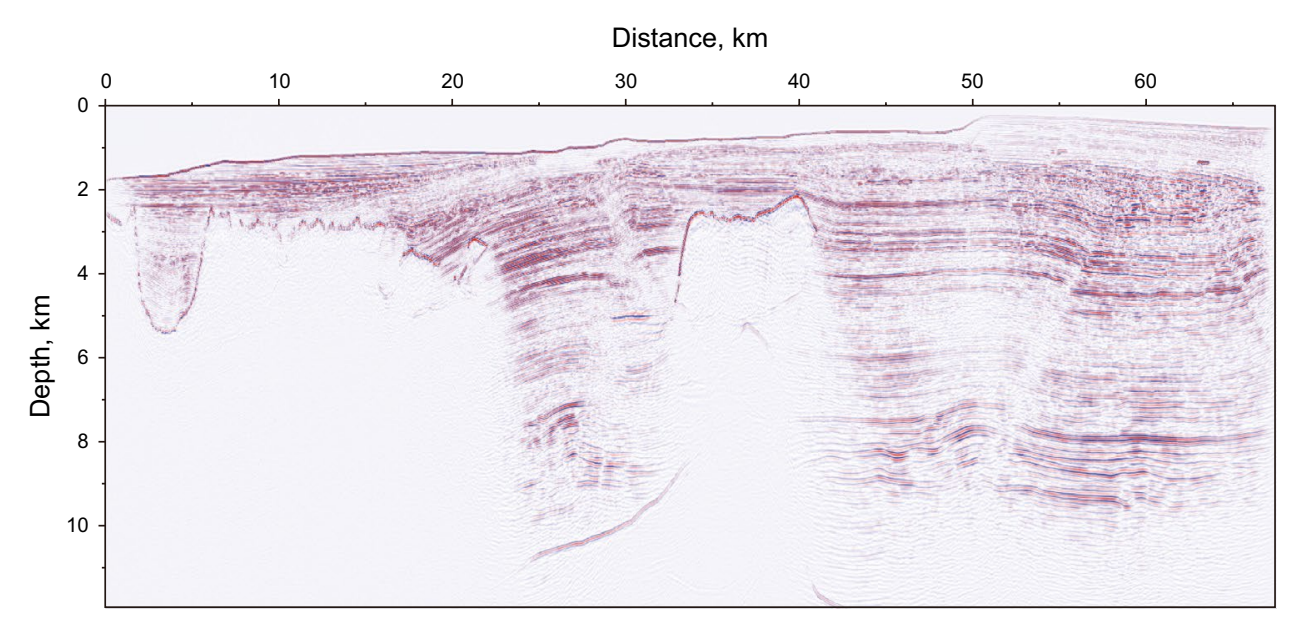

Fig. 12 The GBM result of the BP model using the conventional method

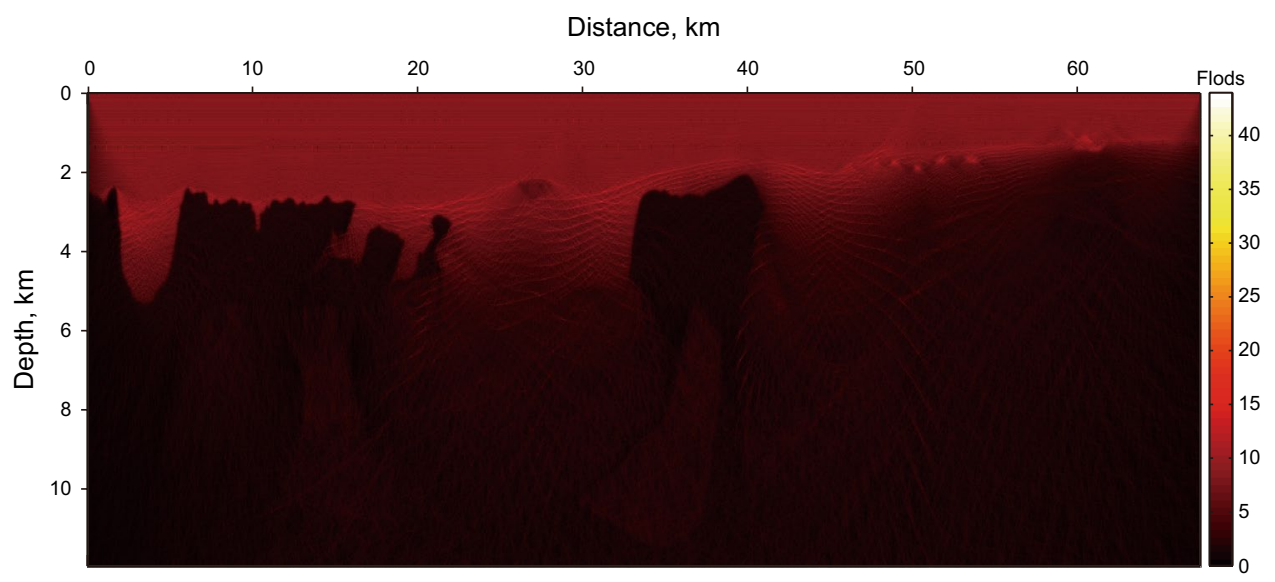

Fig. 13 The ray folds of the subsurface image points in the BP model

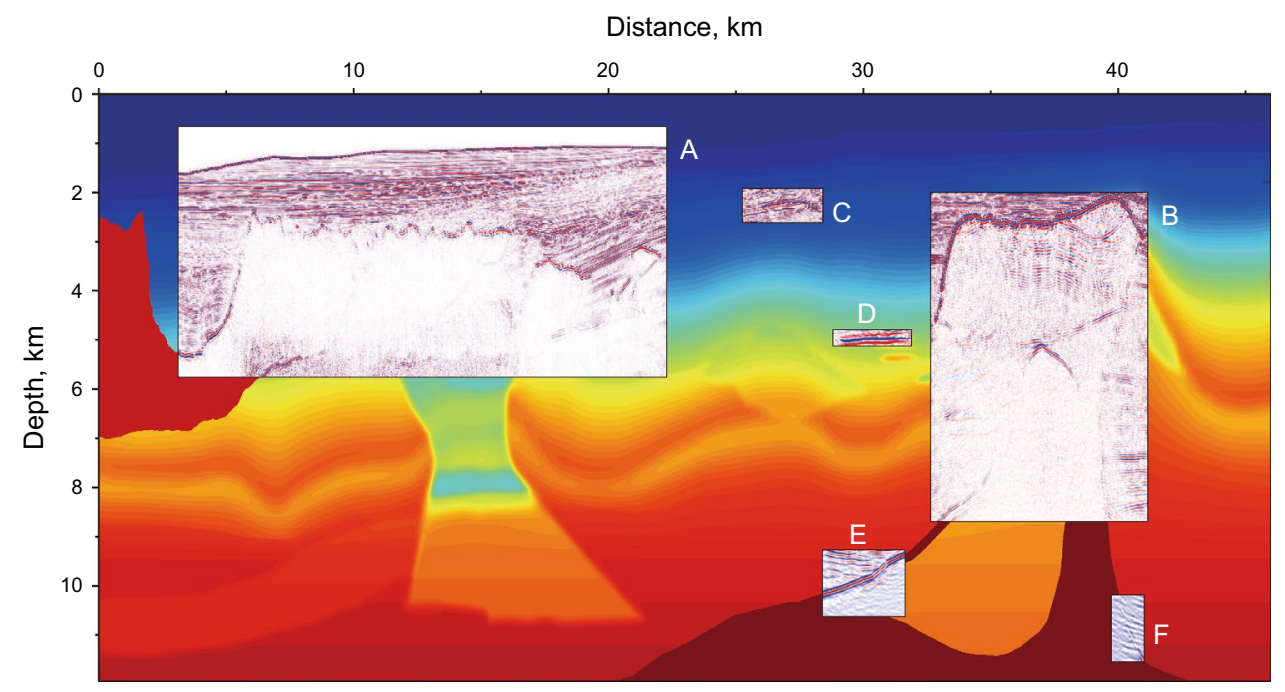

Fig. 14 The target-oriented imaging results of the BP model. Migration results contain a complex rugose multivalued salt body in the left (region A), a deeply rooted salt body in the central part (region B) and four small target regions $(\mathrm{C}, \mathrm{D}, \mathrm{E}$ and F) 

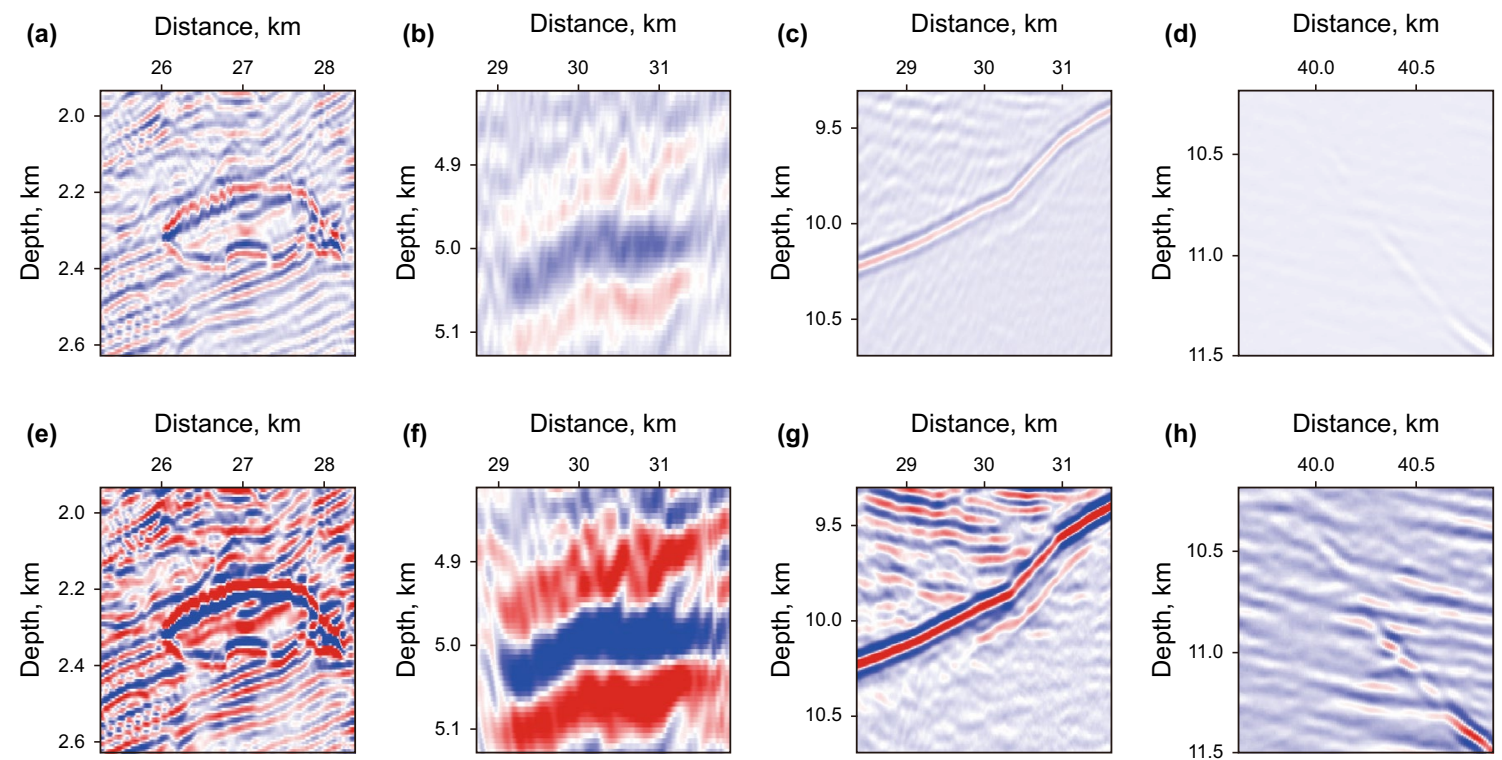

Fig. 15 The comparison of migration results in four small target regions (C, D, E and F). Panels a-d are the magnified views of the migration result (see Fig. 12) using the convention method. Panels $\mathbf{e}-\mathbf{h}$ are the target-oriented imaging results in Fig. 14



Fig. 16 The velocity of the Marmousi model

where the events occur and the model actual interface position are almost same. Figure 15 shows the comparison between the conventional method and our method, and it is obvious that our method restores the structures of six target region better than the conventional method. It should be noted as well that our method is no match for the Popov's method, because Popov's method directly computes time domain Green's function with the help of frequency-domain Gaussian beam and implements a reverse time migration workflow. But our method theoretically has higher computational efficiency for two reasons: (1) without carrying out inverse Fourier transform for each subsurface image point and (2) imaging based the traveltime relationship without calculating and storing the wavefields at multiple time. For more comparison, see also Popov et al. (2010). Meantime, during the numerical experimentation, unexpectedly we discover that our method is still inefficient when an image of a large subsurface volume is required, such as target $\mathrm{A}$ and $\mathrm{B}$ in the BP model, which is an inherent shortcoming of the upgoing ray tracing strategy.

In the fourth example, we carry out migration velocity analysis using our method on the Marmousi model (see Fig. 16). The migration results using different migration velocities are shown in Fig. 17. It is obvious that the accuracy of migration velocity has great influence on the accuracy of image locations. Furthermore, we extract the ADCIGs at $8 \mathrm{~km}$ during the migration procedure to analyze (see Fig. 18). When the migration velocity is too small or too large, the ADCIGs will bend up or down, respectively. In addition, if the migration velocity is accurate and the algorithm is also amplitude-preserved, the ADCIGs can be applied to the AVA and lithological analysis. 
(a)

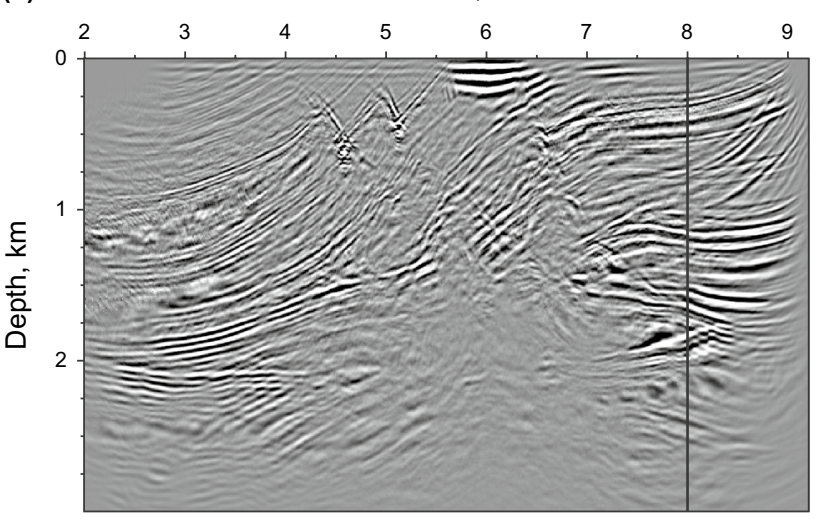

(b)

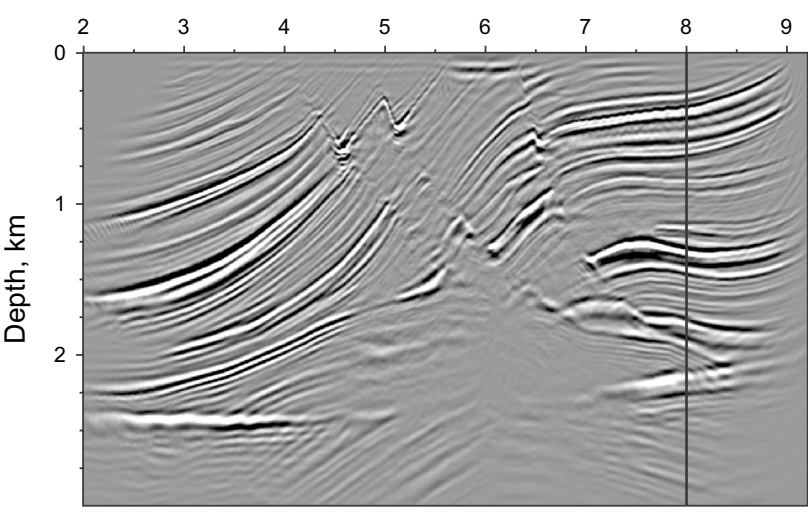

(c)

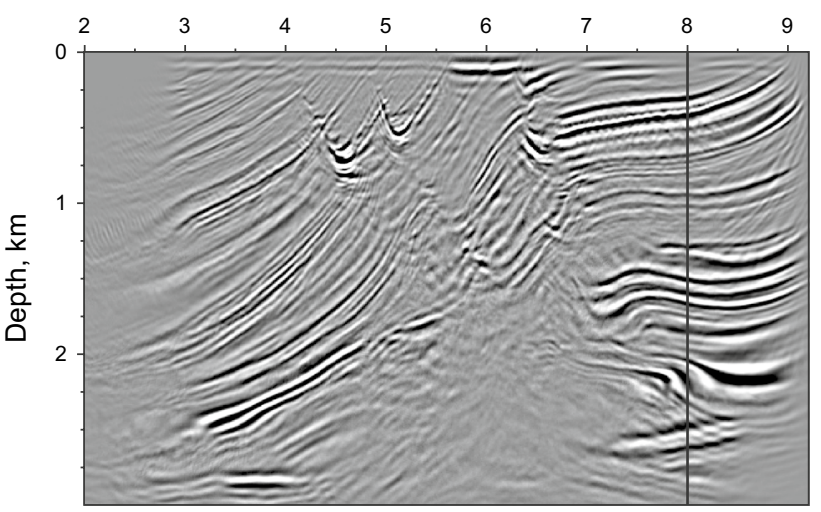

Fig. 17 The migration results of the Marmousi model using different migration velocities: a multiply the true velocity by 0.9 , $\mathbf{b}$ the true velocity, $\mathbf{c}$ multiply the true velocity by 1.1

The final example is the application of a field land data in eastern China by our method, which covers a buried hill drape structure. The velocity model in Fig. 19a is constructed by the traditional ray tomography method and the common-shot gathers is already preprocessed with denoising, muting and bandpass filtering. The 201th shot gather and recording geometry are shown in Fig. 19b, c, respectively. Migration results with the conventional method and our method are depicted in Fig. 20. Compared with the conventional method (see Fig. 20a), our method (see Fig. 20b) produces a clearer image in the shallow layer (marked by red arrows) and makes reflectors more continuous and faults clearer near the buried hill (marked by blue arrows).

\section{Discussion}

In this section, we further discuss our method from two aspects, which are the computational grid and what should be careful when using the proposed method in application, respectively.

As is well known, the computational grid is considerably important for numerical experimentation. When the grid is inappropriate, it will not only affect the accuracy, but also reduce the efficiency. For the conventional method, Hale (1992a) proposed a coarse grid algorithm (see Fig. 21a) considering that the traveltime and amplitude of Gaussian beam vary smoothly within a wavelength range. In this paper, we carry out our method using Hale's trick: first compute the image value in a coarse grid and then interpolate to the refined grid. However, because the migration process of each image point is absolutely independent, Popov et al. (2010) applied an irregular grid of randomly distributed points using a random number generator (see Fig. 21b) to the target-oriented imaging method. They carried out stacking of the different irregular grids and performed interpolation on a regular grid for visualization. Likewise, our method can be implemented on a random grid as well theoretically. In addition, it is possible for us to develop our method to the variable grid (see Fig. 21c, d) for different types of targets in a subsequent study.

When using the proposed method in application, we should be careful about the following aspects: (1) The anti-noise ability of our method is not as good as that of the conventional method for data with low signalto-noise ratio, because the conventional using the local slant-stack approximation can achieve this purpose easily. (2) It should be noted as well that our method is more time-consuming than the conventional method when migration for large subsurface region is required, because upgoing rays need to be shot from every image location in the target region, which will require a huge amount of computations. 

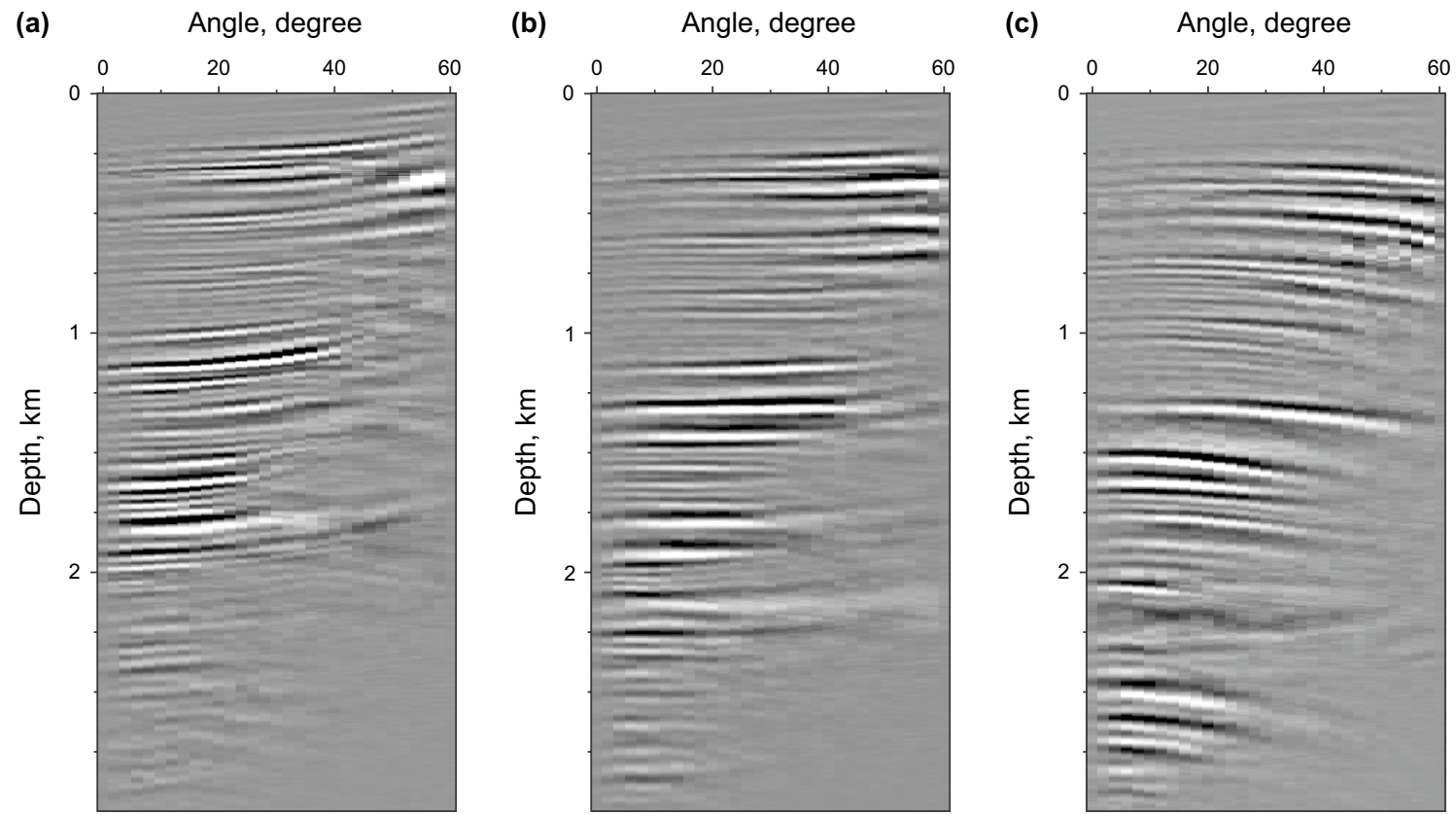

Fig. 18 The ADCIGs at 8 km (marked by black line in Fig. 17) for different migration velocities

(a)

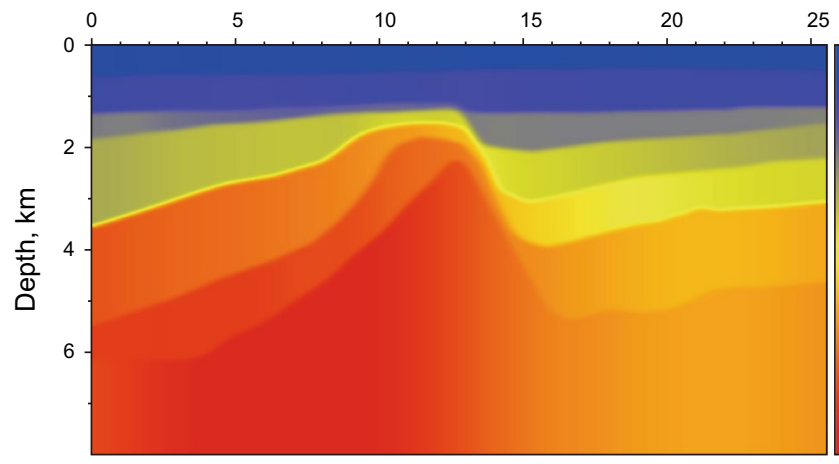

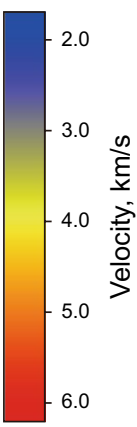

(b)

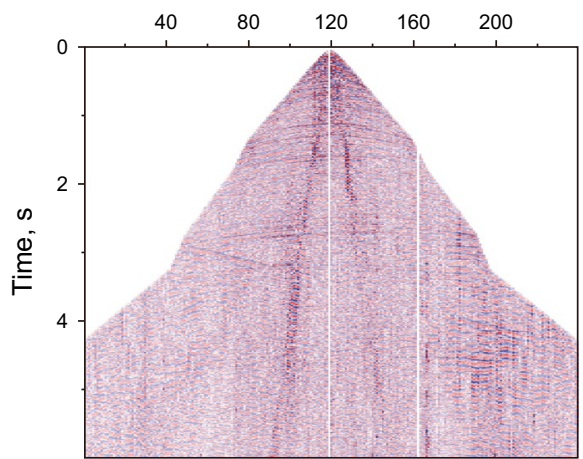

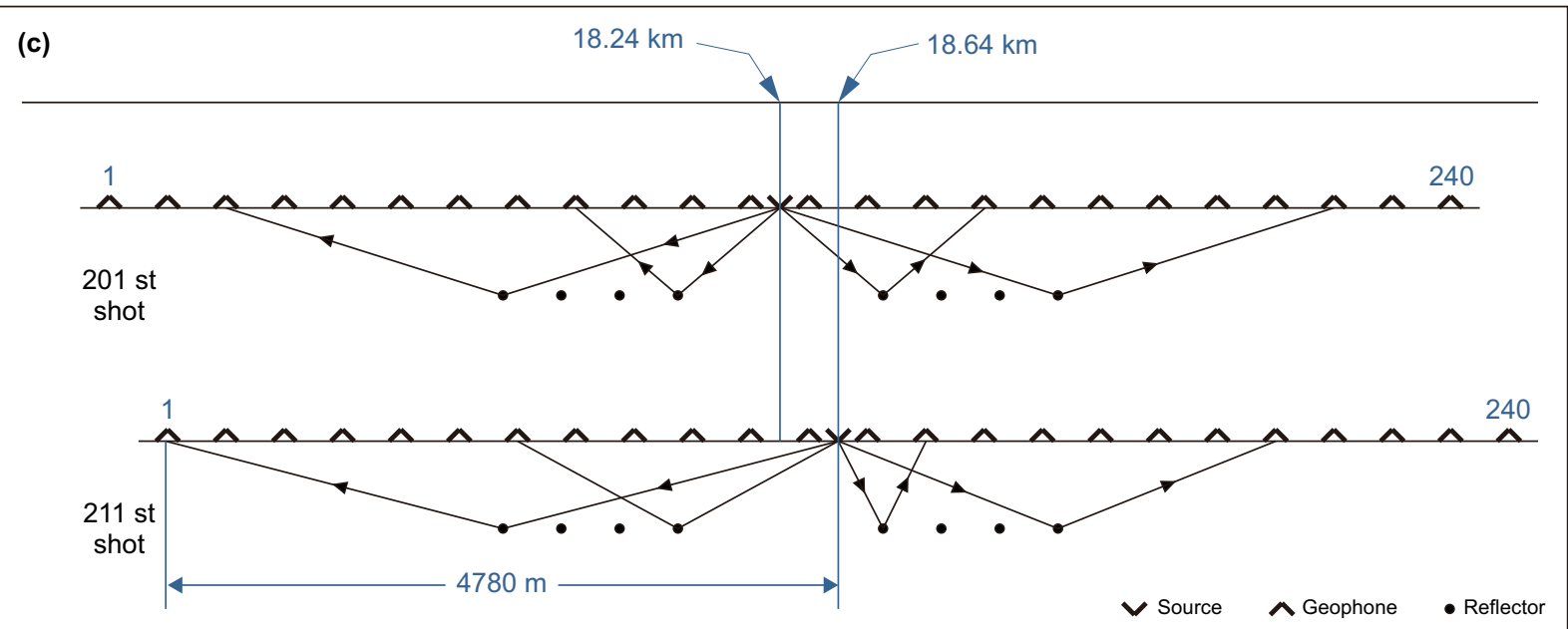

Fig. 19 The land survey in Eastern China: a the built velocity model, $\mathbf{b}$ a preprocessed common-shot gather, $\mathbf{c}$ the recording geometry 
(a)

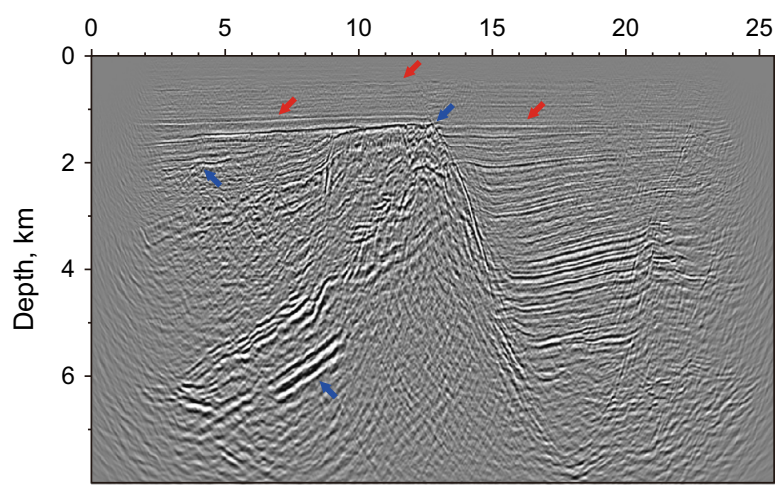

(b)

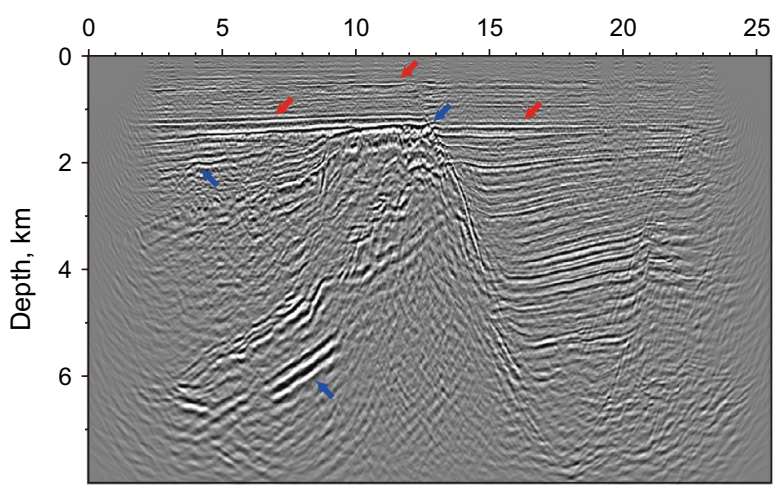

Fig. 20 The migration results for the land survey using: a the conventional method, b our method
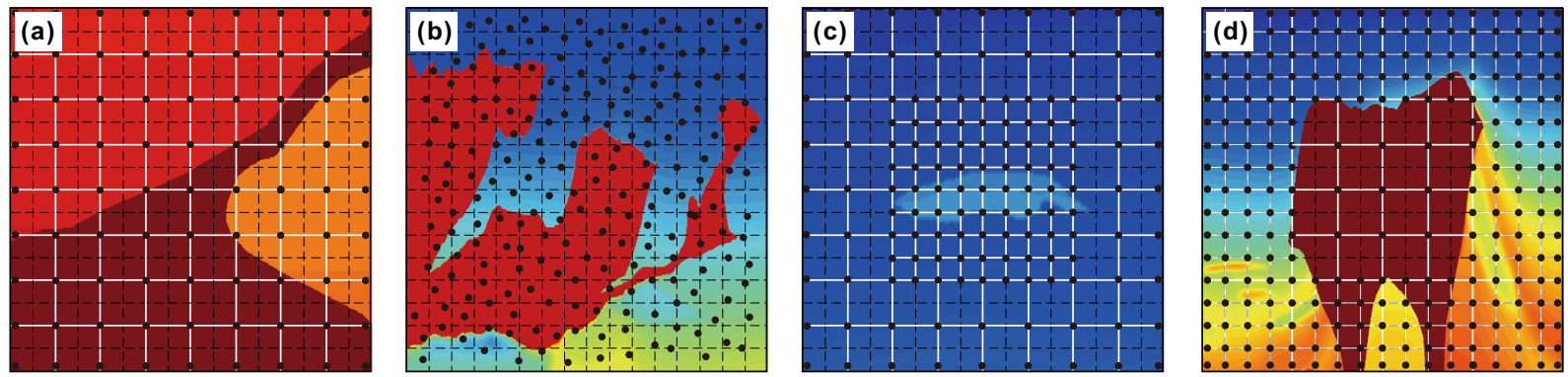

Fig. 21 The different types of computational grid (marked by black dots) and interpolation on a regular and refined grid (divided by the black dashed line) for visualization: a the coarse grid, $\mathbf{b}$ the random grid using a random number generator, $\mathbf{c}, \mathbf{d}$ the variable grid for different types of targets

\section{Conclusion}

To satisfy the requirements of target-oriented imaging, we have provided a target-oriented GBM using a modified ray tracing scheme. Specifically, we modify the conventional Gaussian beam migration scheme, by shooting rays from subsurface image points to receivers to implement wavefield back-propagation. Meantime, considering that computation in frequency domain is particularly inefficiency, we have derived a time domain formula using inverse Fourier transform and Hilbert transform. In addition, making full use of the feature that the imaging process of each subsurface point is absolutely independent, the subdomain parallel algorithm has been applied, which can enhance the computational efficiency significantly. Synthetic and field data examples confirm the validity and the target-oriented imaging capability of our method.

We would like to emphasize the following advantages of our method: the adaptability to the complex target structure and the irregular topography using the variable grid and the random grid; the promising application in industry due to the greater efficiency compared to the wavefield-based GBM. It should be noted as well that our method is more time-consuming than the conventional method when migration for large subsurface region is required.

Furthermore, we would like to draw attention to the fact that our method has high potential for use in the iterative velocity-model-building because the extraction of ADCIGs using our method is extremely handy and efficient. Meantime, amplitude-preserved ADCIGs are of significant importance for the AVA and lithological analysis. In the future, the true amplitude method and the least squares framework could be introduced to improve the fidelity of amplitude.

Open Access This article is distributed under the terms of the Creative Commons Attribution 4.0 International License (http://creativeco mmons.org/licenses/by/4.0/), which permits unrestricted use, distribution, and reproduction in any medium, provided you give appropriate credit to the original author(s) and the source, provide a link to the Creative Commons license, and indicate if changes were made. 


\section{Appendix 1: Derivation of migration formula in time domain}

This appendix gives a detailed derivation process of migration formula in time domain. Here, beginning with Eq. (13), the migration formula in frequency domain after exchanging the order of integration has the following form

$$
\begin{aligned}
I\left(\mathbf{x}_{\mathbf{0}} ; \mathbf{x}_{\mathrm{s}}\right)= & \frac{1}{4 \pi^{2}} \int \mathrm{d} x_{\mathrm{g}} \int_{0}^{2 \pi} \mathrm{d} \theta_{\mathrm{s}} \int_{0}^{2 \pi} \mathrm{d} \theta_{\mathrm{g}} \int i \omega \mathrm{d} \omega \frac{\cos \theta_{\mathrm{s}}}{V_{\mathrm{s}}} \frac{\cos \theta_{\mathrm{g}}}{V_{\mathrm{g}}} \\
& \times A_{\mathrm{s}}^{*} \exp \left[-i \omega T_{\mathrm{s}}^{*}\right] A_{\mathrm{g}}^{*} \exp \left[-i \omega T_{\mathrm{g}}^{*}\right] W\left(\mathbf{x}_{\mathrm{g}} ; \mathbf{x}_{\mathrm{s}} ; \omega\right),
\end{aligned}
$$

here $\cos \theta_{\mathrm{s}} / V_{\mathrm{s}}$ and $\cos \theta_{\mathrm{g}} / V_{\mathrm{g}}$ are the frequency-independent variables and $\int \mathrm{d} \theta_{\mathrm{s}} \int \mathrm{d} \theta_{\mathrm{g}}$ means the integration for each beam. The innermost integration can be expressed as

$I_{1}\left(\mathbf{x}_{\mathbf{0}} ; \mathbf{x}_{\mathbf{s}}\right)=\int i \omega \mathrm{d} \omega A_{\mathrm{s}}^{*} A_{\mathrm{g}}^{*} \exp \left[-i \omega\left(T_{\mathrm{s}}^{*}+T_{\mathrm{g}}^{*}\right)\right] W\left(\mathbf{x}_{\mathrm{g}} ; \mathbf{x}_{\mathrm{s}} ; \omega\right)$,

where this integration can be understood as the crosscorrelation at an image point between the beams emanated from the source and receiver. Hence, we obtain

$I\left(\mathbf{x}_{\mathbf{0}} ; \mathbf{x}_{\mathrm{s}}\right)=\frac{1}{4 \pi^{2}} \int \mathrm{d} x_{\mathrm{g}} \int_{0}^{2 \pi} \mathrm{d} \theta_{\mathrm{s}} \int_{0}^{2 \pi} \mathrm{d} \theta_{\mathrm{g}} \frac{\cos \theta_{\mathrm{s}}}{V_{\mathrm{s}}} \frac{\cos \theta_{\mathrm{g}}}{V_{\mathrm{g}}} I_{1}\left(\mathbf{x}_{\mathbf{0}} ; \mathbf{x}_{\mathrm{s}}\right)$.

Meantime, considering traveltime and amplitude are complex, the integrand is a complex as well. Thus, we first consider the amplitude term of the innermost integration, then we denote

$A_{\mathrm{sg}}(\omega)=A_{\mathrm{s}}^{*}(\omega) A_{\mathrm{g}}^{*}(\omega)$,

$A_{\mathrm{sg}}(\omega)=\operatorname{Re}\left[A_{\mathrm{sg}}(|\omega|)\right]+i \operatorname{sgn}(\omega) \operatorname{Im}\left[A_{\mathrm{sg}}(|\omega|)\right]$,

where Eq. (21) means that the complex amplitude at negative frequency is the complex conjugate of complex amplitude at positive frequency. Furthermore, the complex traveltime is taken into account likewise and we denote

$T_{\mathrm{re}}=\operatorname{Re}\left(T_{\mathrm{s}}^{*}+T_{\mathrm{g}}^{*}\right), A_{\mathrm{re}}=\operatorname{Re}\left[A_{\mathrm{sg}}(|\omega|)\right] ;$

$T_{\mathrm{im}}=\operatorname{Im}\left(T_{\mathrm{s}}^{*}+T_{\mathrm{g}}^{*}\right), A_{\mathrm{im}}=\operatorname{Im}\left[A_{\mathrm{sg}}(|\omega|)\right] ;$

then, inserting Eqs. (21) and (22) into Eq. (19) and rearranging terms, we obtain

$$
\begin{aligned}
I_{1}\left(\mathbf{x}_{\mathbf{0}} ; \mathbf{x}_{\mathbf{s}}\right)= & \int i \omega \mathrm{d} \omega\left(A_{\mathrm{re}}+i \operatorname{sgn}(\omega) A_{\mathrm{im}}\right) \exp \left[-i \omega\left(T_{\mathrm{re}}+i T_{\mathrm{im}}\right)\right] W\left(\mathbf{x}_{\mathbf{g}} ; \mathbf{x}_{\mathbf{s}} ; \omega\right) \\
= & \int i \omega W\left(\mathbf{x}_{\mathbf{g}} ; \mathbf{x}_{\mathbf{s}} ; \omega\right) \exp \left[\omega T_{\mathrm{im}}\right] A_{\mathrm{re}} \exp \left[-i \omega T_{\mathrm{re}}\right] \mathrm{d} \omega \\
& +\int i \omega W\left(\mathbf{x}_{\mathbf{g}} ; \mathbf{x}_{\mathbf{s}} ; \omega\right) \exp \left[\omega T_{\mathrm{im}}\right] i \operatorname{sgn}(\omega) A_{\mathrm{im}} \exp \left[-i \omega T_{\mathrm{re}}\right] \mathrm{d} \omega,
\end{aligned}
$$

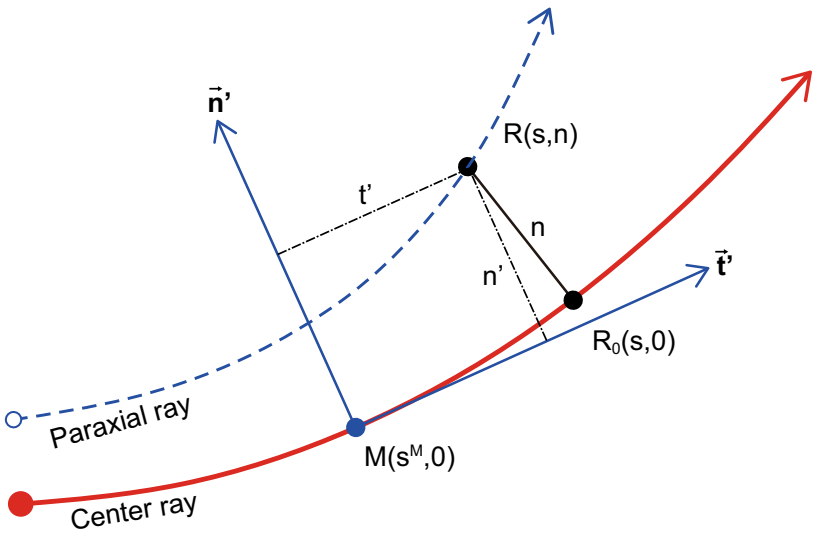

Fig. 22 The propagation of the center ray and the paraxial ray

where $\exp \left[\omega T_{\mathrm{im}}\right]$ must be the attenuation term result from $\operatorname{Im}(P / Q)>0$ in Gaussian beam, which is equivalent to the complex-value filtering for frequency weighted seismic records. Next, we denote

$$
\begin{aligned}
& I_{1}\left(\mathbf{x}_{\mathbf{0}} ; \mathbf{x}_{\mathbf{s}}\right)=I_{2}\left(\mathbf{x}_{\mathbf{0}} ; \mathbf{x}_{\mathbf{s}}\right)+I_{3}\left(\mathbf{x}_{\mathbf{0}} ; \mathbf{x}_{\mathbf{s}}\right), \\
& I_{2}\left(\mathbf{x}_{\mathbf{0}} ; \mathbf{x}_{\mathbf{s}}\right)=\int i \omega W\left(\mathbf{x}_{\mathbf{g}} ; \mathbf{x}_{\mathrm{s}} ; \omega\right) \exp \left[\omega T_{\mathrm{im}}\right] A_{\mathrm{re}} \exp \left[-i \omega T_{\mathrm{re}}\right] \mathrm{d} \omega, \\
& I_{3}\left(\mathbf{x}_{\mathbf{0}} ; \mathbf{x}_{\mathbf{s}}\right)=\int i \omega W\left(\mathbf{x}_{\mathbf{g}} ; \mathbf{x}_{\mathbf{s}} ; \omega\right) \exp \left[\omega T_{\mathrm{im}}\right] \operatorname{sign}(\omega) A_{\mathrm{im}} \exp \left[-i \omega T_{\mathrm{re}}\right] \mathrm{d} \omega .
\end{aligned}
$$

According to Hale (1992b), the first term of Eq. (24) has the form of an inverse Fourier transform $1 / 2 \pi / \exp [-i \omega t]$ and the multiplication of the second term by $-i \operatorname{sgn}(\omega)$ in frequency domain is equivalent to a Hilbert transform in time domain. Therefore, Eq. (23) can be expressed as follows

$I_{1}\left(\mathbf{x}_{\mathbf{0}} ; \mathbf{x}_{\mathrm{s}}\right)=2 \pi A_{\mathrm{re}} \bar{W}\left(\mathbf{x}_{\mathbf{g}} ; \mathbf{x}_{\mathbf{s}} ; T_{\mathrm{re}}, T_{\mathrm{im}}\right)-2 \pi A_{\mathrm{im}} \bar{W}_{\mathrm{H}}\left(\mathbf{x}_{\mathbf{g}} ; \mathbf{x}_{\mathrm{s}} ; T_{\mathrm{re}}, T_{\text {im }}\right)$,

where $\bar{W}$ denotes the inverse Fourier transform of $W$ and subscript $\mathrm{H}$ denotes the Hilbert transform. Finally, inserting Eq. (25) into Eq. (20), we can obtain the migration in time domain

$$
\begin{aligned}
I\left(\mathbf{x}_{\mathbf{0}} ; \mathbf{x}_{\mathrm{s}}\right)= & \frac{1}{2 \pi} \int \mathrm{d} x_{\mathrm{g}} \int_{0}^{2 \pi} \mathrm{d} \theta_{\mathrm{s}} \int_{0}^{2 \pi} \mathrm{d} \theta_{\mathrm{g}} \frac{\cos \theta_{\mathrm{s}}}{V_{\mathrm{s}}} \frac{\cos \theta_{\mathrm{g}}}{V_{\mathrm{g}}} \\
& {\left[A_{\mathrm{re}} \bar{W}\left(\mathbf{x}_{\mathrm{g}} ; \mathbf{x}_{\mathrm{s}} ; T_{\mathrm{re}}, T_{\mathrm{im}}\right)-A_{\mathrm{im}} \bar{W}_{\mathrm{H}}\left(\mathbf{x}_{\mathrm{g}} ; \mathbf{x}_{\mathrm{s}} ; T_{\mathrm{re}}, T_{\mathrm{im}}\right)\right] . }
\end{aligned}
$$

\section{Appendix 2: Extraction of angle domain common image gathers}

As shown in Fig. 22, the complex traveltime at a point $R$ near the center ray satisfies the following approximation (Červený and Pšenčík 2001) 
$\tau(R) \approx \tau^{M}+\frac{\left(s-s^{M}\right)}{v^{M}}-\frac{1}{2} \frac{v_{, s}^{M}\left(s-s^{M}\right)^{2}}{\left(v^{M}\right)^{2}}+\frac{1}{2} \Gamma^{M} n^{2}$,

where $(s, n)$ are ray-centered coordinates, $v^{M}$ and $v_{s}^{M}$ are the velocity and its derivative at a point $M$, respectively; $\Gamma^{M}=P(M) / Q(M), P$ and $Q$ are the dynamic parameters at a point $M$. In order to deduce the form at Cartesian coordinate system of Eq. (27), we introduce the local Cartesian coordinates $\left(t^{\prime}, n^{\prime}\right)$ near the center ray, whose origin of coordinates is a point $M$. According to simple geometric relationship, we can obtain the conversion formula between local Cartesian coordinates and ray-centered coordinates

$n \approx n^{\prime}, s-s^{M} \approx t^{\prime}\left[1-\frac{v_{, n}^{M} n^{\prime}}{v^{M}}\right]$

and inserting Eq. (28) into Eq. (27), we obtain

$\tau(R) \approx \tau^{M}+\frac{t^{\prime}}{v^{M}}-\frac{v_{, n}^{M} t^{\prime} n^{\prime}}{\left(v^{M}\right)^{2}}-\frac{1}{2} \frac{v_{s}^{M}\left(t^{\prime}\right)^{2}}{\left(v^{M}\right)^{2}}+\frac{1}{2} \Gamma^{M}\left(n^{\prime}\right)^{2}$.

Meantime, considering the conversion relationship between Cartesian coordinates and local Cartesian coordinates

$n^{\prime}=\left(x-x^{M}\right) \cos \theta-\left(z-z^{M}\right) \sin \theta$,

$t^{\prime}=\left(x-x^{M}\right) \sin \theta+\left(z-z^{M}\right) \cos \theta$,

and we denote

$$
\begin{aligned}
\boldsymbol{n}^{\prime} & =\left[\begin{array}{l}
n^{\prime} \\
t^{\prime}
\end{array}\right], \boldsymbol{x}=\left[\begin{array}{c}
x-x^{M} \\
z-z^{M}
\end{array}\right], \boldsymbol{l}=\left[\begin{array}{c}
\cos \theta(M) \\
\sin \theta(M)
\end{array}\right], \\
\boldsymbol{\sigma} & =\left[\begin{array}{cc}
\cos \theta(M) & -\sin \theta(M) \\
\sin \theta(M) & \cos \theta(M)
\end{array}\right],
\end{aligned}
$$

where $\theta$ is the center ray propagation angle and $\sigma$ is the rotation matrix. Hence, Eq. (30) can be written as $n^{\prime}=\sigma x$ and further considering $\left(\boldsymbol{n}^{\prime}\right)^{\mathrm{T}}=\boldsymbol{x}^{\mathrm{T}} \sigma^{\mathrm{T}}$ (T denotes the matrix transposition), Eq. (29) can be simplified to the following form

$\tau(R) \approx \tau^{M}+\frac{\boldsymbol{l}^{\mathrm{T}} \boldsymbol{x}}{v^{M}}+\frac{1}{2} \boldsymbol{x}^{\mathrm{T}} \mathbf{W} \boldsymbol{x}$,

here

$$
\begin{aligned}
& \mathbf{W}=\boldsymbol{\sigma}^{\mathrm{T}} \mathbf{A} \boldsymbol{\sigma}, \mathbf{A}=\left(v^{M}\right)^{-2}\left[\begin{array}{cc}
\left(v^{M}\right)^{2} \Gamma^{M} & -v_{, n}^{M} \\
-v_{, n}^{M} & -v_{, s}^{M}
\end{array}\right], \\
& v_{, n}=v_{, x} \cos \theta-v_{, z} \sin \theta, v_{, s}=v_{, x} \sin \theta+v_{, z} \cos \theta .
\end{aligned}
$$

According to Eq. (32), the real-valued traveltime of a point $R$ at the paraxial ray can be expressed as follows

$$
\begin{aligned}
\tau(R)= & \tau(M)+\left[p_{x}^{M}\left(x^{R}-x^{M}\right)+p_{z}^{M}\left(z^{R}-z^{M}\right)\right] \\
& +\frac{1}{2}\left[\begin{array}{c}
x^{R}-x^{M} \\
z^{R}-z^{M}
\end{array}\right] \cdot \operatorname{Re}(\mathbf{W}) \cdot\left[x^{R}-x^{M} z^{R}-z^{M}\right],
\end{aligned}
$$

where $p_{x}^{M}$ and $p_{z}^{M}$ are the horizontal and vertical slowness, respectively, and $\operatorname{Re}(\mathbf{W})$ denotes the real part of complex matrix W. Next, we take the derivative of Eq. (34) along the $x$ direction and obtain

$\frac{\partial \tau(R)}{\partial x}=p_{x}^{M}+\operatorname{Re}\left(\mathrm{W}_{11}\right)\left(x^{R}-x^{M}\right)+\operatorname{Re}\left(\mathrm{W}_{12}\right)\left(z^{R}-z^{M}\right)$,

here $\mathrm{W}_{11}$ and $\mathrm{W}_{12}$ are the top-left and top-right elements of matrix $\mathbf{W}$, then considering $p_{x}^{R}=\partial \tau(R) / \partial x$, we obtain

$p_{x}^{R}=p_{x}^{M}+\operatorname{Re}\left(\mathrm{W}_{11}\right)\left(x^{R}-x^{M}\right)+\operatorname{Re}\left(\mathrm{W}_{12}\right)\left(z^{R}-z^{M}\right)$.

Similarly, we can deduce the formula along the $z$ direction

$p_{z}^{R}=p_{z}^{M}+\operatorname{Re}\left(\mathrm{W}_{21}\right)\left(x^{R}-x^{M}\right)+\operatorname{Re}\left(\mathrm{W}_{22}\right)\left(z^{R}-z^{M}\right)$,

then the propagation angle of a point $R$ at the paraxial ray can be calculated by the following formula

$\alpha_{\mathrm{s}}= \begin{cases}-\pi+\arctan \left(p_{x}^{R} / p_{z}^{R}\right), & p_{x}^{R}<0, p_{z}^{R}<0 ; \\ \pi+\arctan \left(p_{x}^{R} / p_{z}^{R}\right), & p_{x}^{R}>0, p_{z}^{R}<0 ; \\ \arctan \left(p_{x}^{R} / p_{z}^{R}\right), & \text { others. }\end{cases}$

(See Fig. 22).

As shown in Fig. 23, $\mathbf{x}_{\mathbf{s}}$ and $\mathbf{x}_{\mathbf{g}}$ are the source and geophone location, respectively; $\mathbf{x}_{\mathbf{0}}$ is the subsurface image point; $\theta$ is the incident angle of the source ray, i.e., the reflection angle with respect to the normal; $\hat{\alpha}_{\mathrm{s}}$ and $\hat{\alpha}_{\mathrm{g}}$ are unit vectors along the rays from source and image point, respectively; $\hat{v}$ is the dip vector, which is perpendicular to the reflector and $v$ is the migration dip; $\alpha_{\mathrm{s}}$ is the propagation angle of the source ray which can be obtained by Eq. (38), and $\alpha_{\mathrm{g}}$ is the initial angle of the upgoing ray tracing. In order to extract the ADCIGs, we merely need to calculate the opening angle at the image point $\mathbf{x}_{\mathbf{0}}$ and place the migrated amplitude into the position of ADCIGs according to that opening angle. According to simple angle relationship, the opening angle formula can be expressed as follows

$\theta=\frac{1}{2}\left[\pi-\left(\alpha_{\mathrm{g}}-\alpha_{\mathrm{s}}\right)\right]$.

(See Fig. 23). 


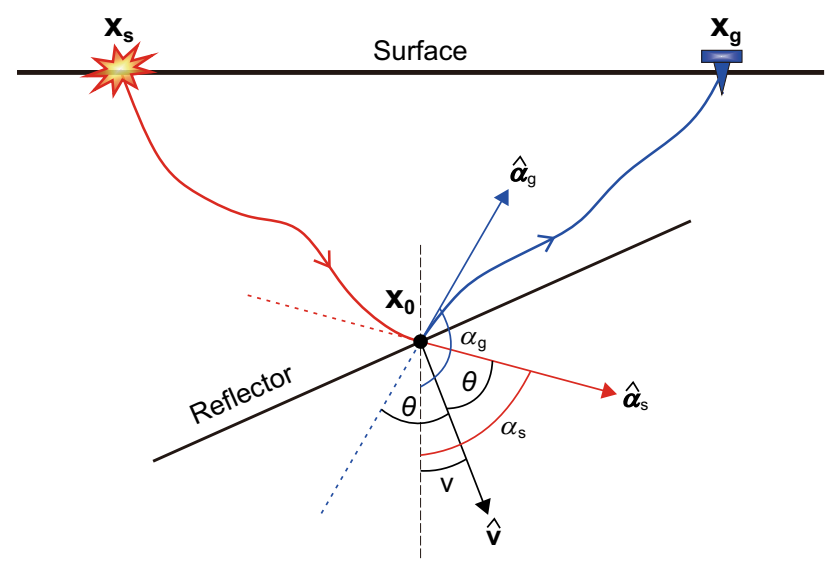

Fig. 23 Sketch of the local angle domain

\section{References}

Albertin U, Yingst D, Kitchenside P, et al. True-amplitude beam migration. In: 74th annual international meeting, SEG, expanded abstracts. 2004. p. 949-52.

Alkhalifah T. Gaussian beam depth migration for anisotropic media. Geophysics. 1995;60:1474-84.

Babich VM, Popov MM. Gaussian summation method. Radiophys Quantum Electron. 1989;32(12):1063-81.

Bai M, Chen XH, Wu J, et al. Multiple-component Gaussian beam resverse-time based on attenuation. Chin J Geophys. 2016;59(9):3379-93 (in Chinese).

Billette FJ, Brandsberg-Dahl S. The 2004 BP velocity benchmark. In: 67 th annual international meeting, EAGE, extended abstracts. 2005. p. B035.

Bleistein N. Hagedoorn told us how to do Kirchhoff migration and inversion. Lead Edge. 1999;18(8):918-27.

Casasanta L, Gray SH. Converted-wave beam migration with sparse sources or receivers. Geophys Prospect. 2015;63(3):534-51.

Červený V. Synthetic body wave seismograms for laterally varying layered structures by the Gaussian beam method. Geophys J Int. 1983;73(2):389-426.

Červený V, Pšenčík I. Gaussian beams in two-dimensional elastic inhomogeneous media. Geophys J Int. 1983a;72(2):417-33.

Červený V, Pšenčík I. Gaussian beams and paraxial ray approximation in three-dimensional elastic inhomogeneous media. J Geophys. 1983b;53(1):1-15.

Červený V, Pšenčík I. Gaussian beams in elastic 2-D laterally varying layered structures. Geophys J Int. 1984;78(1):65-91.

Červený V, Popov MM, Pšenčík I. Computation of wave fields in inhomogeneous media-Gaussian beam approach. Geophys J Int. 1982;70(1):109-28.

Červený V, Pšenčík I. Seismic ray theory. Encycl Earth Sci. 2001;4(Supplement S0):93-105.

Gao C, Sun JG, Qi P, et al. 2-D Gaussian-beam migration of commonshot records in time domain. Chin J Geophys. 2015;58(4):133340 (In Chinese).

Gray SH. Gaussian beam migration of common-shot records. Geophysics. 2005;70(4):S71-7.

Gray SH, Bleistein N. True-amplitude Gaussian-beam migration. Geophysics. 2009;74(2):S11-23.

Gray SH, Notfors C, Bleistein N. Imaging using multi-arrivals: Gaussian beams or multi-arrival Kirchhoff? In: 72nd annual international meeting, SEG, expanded abstracts. 2002. p. 1117-20.
Hale D. Computational aspects of Gaussian beam migration. Colorado School of Mines Center for Wave Phenomena Report. 1992a. p. 139.

Hale D. Migration by the Kirchhoff, slant stack, and Gaussian beam methods. Colorado School of Mines Center for Wave Phenomena Report. 1992b. p. 121.

Hill NR. Gaussian beam migration. Geophysics. 1990;55(11):1416-28.

Hill NR. Prestack Gaussian-beam depth migration. Geophysics. 2001;66(4):1240-50.

$\mathrm{Hu}$ H, Liu Y, Zheng Y, et al. Least-squares Gaussian beam migration. Geophysics. 2016;81(3):S87-100.

Huang JP, Yuan ML, Zhang Q, et al. Reverse time migration with elastodynamic Gaussian beams. J Earth Sci. 2017;28(4):695-702.

Kachalov AP, Popov MM. Application of the method of summation of Gaussian beams for calculation of high-frequency wave fields. Sov Phys Dokl. 1981;26:604-6.

Li ZC, Liu Q, Han WG, et al. Angle domain converted wave Gaussian beam migration in VTI media. Chin J Geophys. 2018;61(4):147181 (in Chinese).

Liu J, Palacharla G. Multiarrival Kirchhoff beam migration. Geophysics. 2011;76(5):WB109-18.

Liu W, Wang Y. Target-oriented reverse time migration for two-way prestack depth imaging. In: 78th annual international meeting, SEG, expanded abstracts. 2008. p. 2326-30.

Nowack RL, Sen MK, Stoffa PL. Gaussian beam migration for sparse common-shot and common-receiver data. In: 73rd annual international meeting, SEG, expanded abstracts. 2003. p. 1114-7.

Popov MM. Novuy method vutchislenya volnovuh poley v vusokochastotnom pribligenii. Zapiski Nauchnyh Seminarov LOMI. 1981;104:195-216.

Popov MM. A new method of computation of wave fields using Gaussian beams. Wave Motion. 1982;4(1):85-97.

Popov MM. Ray theory and Gaussian beam method for geophysicists. Salvador: EDUFBA; 2002

Popov MM, Semtchenok NM, Popov PM, et al. Reverse time migration with Gaussian beams and its application to a few synthetic data sets. In: 77th annual international meeting, SEG, expanded abstracts. 2007. p. 2165-9.

Popov MM, Semtchenok NM, Popov PM, et al. Reverse time migration with Gaussian beams and velocity analysis applications. In: 70th annual international meeting, EAGE, extended abstracts. 2008. p. F048.

Popov MM, Semtchenok NM, Popov PM, et al. Depth migration by the Gaussian beam summation method. Geophysics. 2010;75(2):S81-93.

Protasov M. 2-D Gaussian beam imaging of multicomponent seismic data in anisotropic media. Geophys J Int. 2015;203(3):2021-31.

Sava PC, Fomel S. Angle-domain common-image gathers by wavefield continuation methods. Geophysics. 2003;68(3):1065-74.

Sava P, Fomel S. Coordinate-independent angle-gathers for wave equation migration. In: 75th annual international meeting, SEG, expanded abstracts. 2005. p. 2052-5.

Sava P, Fomel S. Time-shift imaging condition in seismic migration. Geophysics. 2006;71(6):S209-17.

$\mathrm{Xu} \mathrm{S}$, Chauris $\mathrm{H}$, Lambaré $\mathrm{G}$, et al. Common-angle migration: a strategy for imaging complex media. Geophysics. 2001;66(6):1877-94.

Yang J, Zhu H. A practical data-driven optimization strategy for Gaussian beam migration. Geophysics. 2017;83(1):S81-92.

Yang J, Zhu H, Huang J, et al. 2D isotropic elastic Gaussian beam migration for common-shot multicomponent records. Geophysics. 2017;83(2):S127-40.

Yang J, Zhu H, McMechan G, et al. Time-domain least-squares migration using the Gaussian beam summation method. Geophys J Int. 2018;214(1):548-72. 
Yue YB, Li ZC, Zhang P, et al. Prestack Gaussian beam depth migration under complex surface conditions. Appl Geophys. 2010;7(2):143-8.

Yue YB, Li ZC, Qian ZP, et al. Amplitude-preserved Gaussian beam migration under complex topographic conditions. Chin J Geophys. 2012;55(4):1376-83 (in Chinese).
Zhang Y, Xu S, Bleistein N, et al. True-amplitude, angle-domain, common-image gathers from one-way wave-equation migrations. Geophysics. 2007;72(1):S49-58.

Zhu T, Gray SH, Wang D. Prestack Gaussian-beam depth migration in anisotropic media. Geophysics. 2007;72(3):S133-8. 